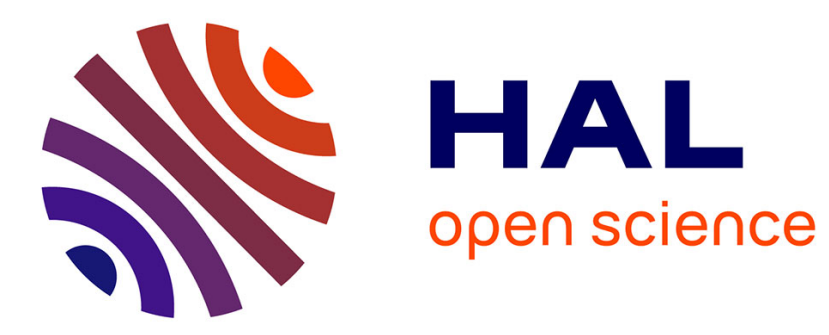

\title{
Nonlinear Conditioning Circuits for Piezoelectric Energy Harvesters
}

\author{
Adrien Badel, Elie Lefeuvre
}

\section{To cite this version:}

Adrien Badel, Elie Lefeuvre. Nonlinear Conditioning Circuits for Piezoelectric Energy Harvesters. Nonlinearity in Energy Harvesting Systems, 25 (2), Springer International Publishing, pp.321-359, 2016, 10.1007/978-3-319-20355-3 . hal-02042560

\section{HAL Id: hal-02042560 https://hal.science/hal-02042560}

Submitted on 3 Jan 2020

HAL is a multi-disciplinary open access archive for the deposit and dissemination of scientific research documents, whether they are published or not. The documents may come from teaching and research institutions in France or abroad, or from public or private research centers.
L'archive ouverte pluridisciplinaire HAL, est destinée au dépôt et à la diffusion de documents scientifiques de niveau recherche, publiés ou non, émanant des établissements d'enseignement et de recherche français ou étrangers, des laboratoires publics ou privés. 


\section{Nonlinear Conditioning Circuits for Piezoelectric Energy Harvesters}

Adrien Badel, SYMME, Université Savoie Mont Blanc, F74000 Annecy, France

Elie Lefeuvre, IEF, Université Paris Sud, F91405 Orsay cedex, France

\section{INTRODUCTION}

Design and analysis of piezoelectric vibration energy harvesters is a complex multi-physics problem related to mechanics, materials science and electronics. The analysis of works published in the field of piezoelectric energy harvesting over the last decade shows that numerous papers focused on mechanical optimization without taking into account the actual constraints and requirements on the electrical side of the systems (i.e. the electric circuit was often modeled as a simple resistor). Conversely, other works aimed at optimizing systems from the electrical point of view without taking into account the mechanical effects induced by the energy conversion process. Consequently, in both cases the solutions proposed were not truly optimal or remained very far from practical applications.

To highlight the main aspects of this multi-physics problem, this chapter begins with general considerations about harvested power based on the simple and well-known model proposed by William and Yates [1]. Starting from this model, the maximal power and the frequency bandwidth of the system is analyzed, and a figure of merit taking into account both the power and the bandwidth of the energy harvester is proposed.

This model is then refined to include the description of piezoelectric electromechanical coupling, leading to accurate and reliable behavioral representation of most linear inertial piezoelectric vibration energy harvesters.

Using the classical analogies between electrical and mechanical figures, an equivalent electrical circuit representing the whole electromechanical system is described. Such circuit may be very convenient to study the system associated to its electronic interface using SPICE-type simulations. In order to get the model parameters representing an actual vibration energy harvester, an identification procedure based on the measurement of the complex admittance is presented.

The performances of the system using optimal impedance matching approach is then analyzed, followed by a brief outline of a possible implementation using a PWM inverter circuit.

The association of the piezoelectric vibration energy harvester with the classical rectifier circuit is then analyzed, showing the need for an additional maximum power point tracking system.

After a short description of the main nonlinear interface circuits developed over the last decade, a detailed analysis of the so-called OSECE interface circuit is exposed.

Finally, this chapter discusses the possibility of tuning the resonant frequency of the piezoelectric energy harvester through its interface circuit. The theoretical analysis of a new interface developed for this purpose and termed FTSECE is presented. 


\section{A FIRST VERY SIMPLE MODEL FOR KINETIC ENERGY HARVESTERS}

The power generated by a vibration energy harvester depends on the transducer used for the electromechanical energy conversion and on the way the transducer is implemented in a mechanical structure that is suitable to capture ambient mechanical energy.

In most vibration energy harvesters (VEH) an inertial mass driven by the ambient acceleration is used to transmit mechanical energy to the electromechanical transducer. A simple \{mass-springdamper\} system, initially proposed in [1] can be used to model this behavior. A schematic of such an inertial vibration energy harvester is shown in Figure 1 , where $D_{L}$ is a damper that embodies mechanical and electrical losses and $D_{H}$ is a damper that corresponds to the electromechanical transducer. The assumption of modeling the transducer by a simple damper is valid in the case of sinusoidal motion of the inertial mass, choosing the value of $D_{H}$ such as the energy dissipated into the damper during one mechanical period corresponds to the harvested energy.

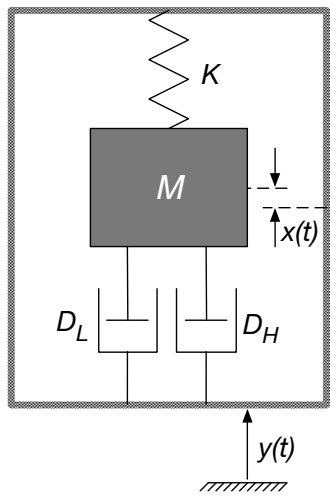

Figure 1. Inertial vibration energy harvester

\subsection{Consideration on the harvested power}

The equation governing the motion of the inertial mass is given by (1.1), where $\gamma=\ddot{y}$ is the ambient acceleration.

$$
M \ddot{x}+\left(D_{H}+D_{L}\right) \dot{x}+K x=-M \gamma
$$

The relative motion $x$ of the mass with respect to the housing can then be expressed in the frequency domain as:

$$
\underline{x}=-\frac{\frac{\gamma}{\omega_{0}^{2}}}{\left(1-\Omega^{2}\right)+2 j \Omega\left(\xi_{L}+\xi_{H}\right)} \text { with }\left\{\begin{array}{l}
\omega_{0}=\sqrt{\frac{K}{M}} \\
\xi_{L}=\frac{D_{L}}{2} \sqrt{\frac{1}{K M}} \\
\xi_{H}=\frac{D_{H}}{2} \sqrt{\frac{1}{K M}} \\
\Omega=\frac{\omega}{\omega_{0}}
\end{array}\right.
$$

Where $\omega$ is the operation angular frequency, $\omega_{0}$ is the mechanical oscillator natural angular frequency, $\Omega$ is the normalized operation frequency and $\xi_{L}$ and $\xi_{H}$ are the damping ratios due to intrinsic losses and energy harvesting respectively.

Normalizing $x$ with respect to $\gamma_{m} / \omega_{0}^{2}$ (which is the ambient displacement magnitude) leads to: 


$$
\underline{x}^{\prime}=-\frac{1}{\left(1-\Omega^{2}\right)+2 j \Omega\left(\xi_{L}+\xi_{H}\right)}
$$

The harvested power, calculated as the power dissipated in the damper $D_{H}$ is then:

$$
P=\frac{D_{H} \omega^{2} x_{M}^{2}}{2}=\frac{\xi_{H} \Omega^{2}}{\left(1-\Omega^{2}\right)^{2}+4 \Omega^{2}\left(\xi_{L}+\xi_{H}\right)^{2}} \frac{M \gamma_{M}^{2}}{\omega_{0}}
$$

This power reaches a maximum at the resonant frequency $(\Omega=1)$ and when the damping ratios are equals $\left(\xi_{H}=\xi_{L}\right)$ :

$$
P_{\lim }=\frac{D_{H} \omega^{2} x_{M}^{2}}{2}=\frac{M \gamma_{M}^{2}}{16 \omega_{0} \xi_{L}}
$$

The maximal power that can be harvested is proportional to the inertial mass and to the square of the ambient acceleration magnitude. It is also inversely proportional to the losses (mechanical and electrical) in the harvester and to the natural angular frequency.

This power limit is independent from the transduction principle itself. This means that whatever the transduction mechanism, the maximal power can be harvested provided that the electromechanical coupling is high enough to reach the intrinsic damping ratio [2].

The harvested power can be normalized as shown in (1.6). This allows to compare the performances of different energy harvesters independently from their resonant frequencies inertial masses and from the ambient acceleration magnitude. The normalized power cannot exceed $1 /\left(2 \xi_{L}\right)=Q_{M}$ which is the quality factor of the mechanical oscillator.

$$
P^{\prime}=P \frac{8 \omega_{0}}{M \gamma_{M}^{2}}=\frac{8 \xi_{H} \Omega^{2}}{\left(1-\Omega^{2}\right)^{2}+4 \Omega^{2}\left(\xi_{L}+\xi_{H}\right)^{2}}
$$

It is worthy of note that this result is only valid for inertial linear vibration energy harvesters driven around their resonance frequencies by sinusoidal vibrations.

\subsection{Consideration on the frequency bandwidth}

When considering an inertial vibration energy harvester, not only the maximal power but also the frequency bandwidth has to be studied. Equation (1.5) shows that intrinsic losses represented by $\xi_{L}$ should be minimized to increase the maximal power. The goal of this subsection is to highlight the effect of $\xi_{L}$ on the frequency response of the VEH.

We consider here the case in which the damping ratio due to energy harvesting $\xi_{H}$ is equal to $\xi_{L}$ in order to maximize the power at the resonance frequency. In a first consideration, $\xi_{H}$ is kept constant over the frequency. The normalized power is then given by:

$$
P^{\prime}=\frac{8 \xi_{L} \Omega^{2}}{\left(1-\Omega^{2}\right)^{2}+16 \Omega^{2} \xi_{L}^{2}}
$$

We define the angular frequency bandwidth $\Delta \omega$ as the angular frequency range where the power is at least $50 \%$ of its maximal value $\left(P_{\lim }^{\prime}=Q_{M}\right)$. It is obtained seeking $\Omega_{1}$ and $\Omega_{2}$ so that $P^{\prime}\left(\Omega_{1}\right)=P^{\prime}\left(\Omega_{2}\right)=P_{\max }^{\prime} / 2$. The normalized bandwidth $\Delta \Omega$ is then given by (1.8), and the bandwidth by $\Delta \omega=\Delta \Omega \cdot \omega_{0}$. 


$$
\left\{\begin{array}{l}
\Omega_{1}=\sqrt{8 \xi_{L}^{2}+1-4 \xi_{L} \sqrt{4 \xi_{L}^{2}+1}} \\
\Omega_{2}=\sqrt{8 \xi_{L}^{2}+1+4 \xi_{L} \sqrt{4 \xi_{L}^{2}+1}}
\end{array} \Rightarrow \Delta \Omega=\Omega_{2}-\Omega_{1}=4 \xi_{L}\right.
$$

Figure 2 shows the normalized power as a function of the frequency, for three different values of the intrinsic losses. It is clearly shown that decreasing the intrinsic losses increases the maximal power but decreases the bandwidth.

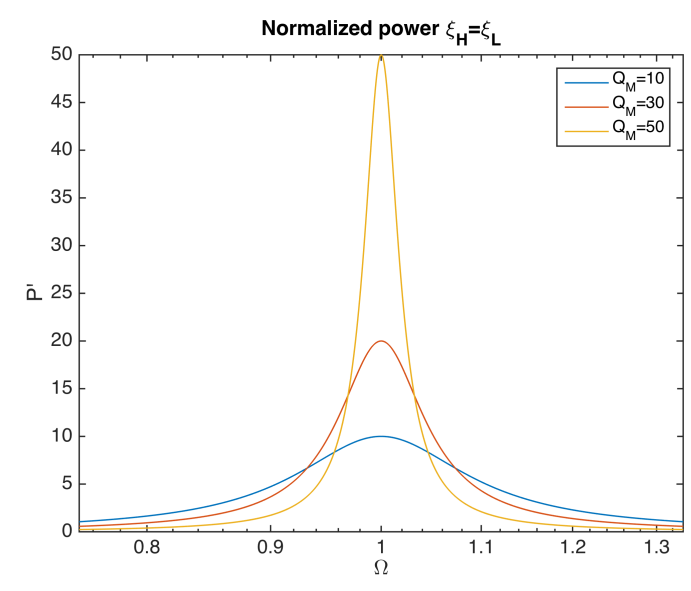

Figure 2. Normalized power vs. frequency for different values of $Q_{M}=1 /\left(2 \xi_{L}\right)$ when $\xi_{H}=\xi_{L}$

It is possible to increase the bandwidth if $\xi_{H}$ can be adjusted as a function of the frequency. An ideal case is now considered, where $\xi_{H}$ can be tuned without any limitation. The optimal value of $\xi_{H}$ that maximizes the harvested power can be obtained as a function of $\Omega$ by looking for the roots of the derivative of Eq. (1.6) with respect to $\xi_{H}$. It is given by:

$$
\xi_{\text {Hopt }}=\frac{\sqrt{\Omega^{4}+4 \Omega^{2} \xi_{L}^{2}-2 \Omega+1}}{2 \Omega}
$$

And the expression of the normalized power is then:

$$
P^{\prime}\left(\xi_{\text {Hopt }}\right)=\frac{4 \Omega \sqrt{\Omega^{4}+4 \Omega^{2} \xi_{L}^{2}-2 \Omega+1}}{4 \Omega^{2}\left(\xi_{L}+\frac{\sqrt{\Omega^{4}+4 \Omega^{2} \xi_{L}^{2}-2 \Omega+1}}{2 \Omega}\right)^{2}+\left(\Omega^{2}-1\right)^{2}}
$$

The new expressions of the half-power normalized angular frequencies are given by (1.11), together with the normalized bandwidth, which is shown to be $\sqrt{2}$ times larger than the case where $\xi_{H}$ is kept equal to $\xi_{L}$.

$$
\left\{\begin{array}{l}
\Omega_{1}=\sqrt{16 \xi_{L}^{2}+1-4 \xi_{L} \sqrt{16 \xi_{L}^{2}+2}} \\
\Omega_{2}=\sqrt{16 \xi_{L}^{2}+1+4 \xi_{L} \sqrt{16 \xi_{L}^{2}+2}}
\end{array} \Rightarrow \Delta \Omega=\Omega_{2}-\Omega_{1}=4 \sqrt{2} \xi_{L}\right.
$$

An interesting result is shown here: whatever the strategy used to tune $\xi_{H}$, the bandwidth is proportional to $\xi_{L}$, whereas the maximal power is inversely proportional to it.

Figure 3 exhibits the normalized power and the optimal value of $\xi_{H}$ as a function of the frequency, for different values of $Q_{M}=1 /\left(2 \xi_{L}\right)$. It is shown that the bandwidth can be enlarged provided that $\xi_{H}$ can be adequately increased while $\Omega$ gets away from 1 . 

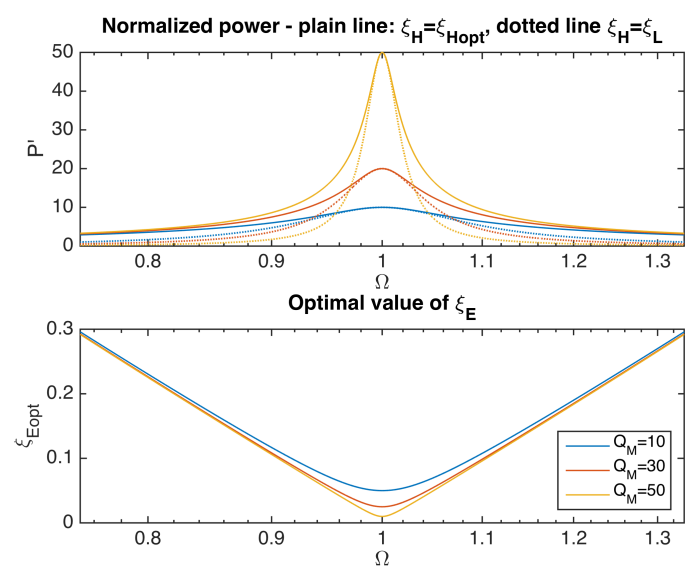

Figure 3. Upper plot: normalized power vs. frequency for different values of $Q_{M}=1 /\left(2 \xi_{L}\right)$ when $\xi_{H}=\xi_{\text {Hopt }}(\Omega)$ (plain line) and when $\xi_{H}=\xi_{L}$ (dotted line), Lower plot: optimal value of $\xi_{H}$

\subsection{Figure of merit}

An ideal VEH would exhibit a large output power over a wide frequency range. For a given VEH, a relevant figure of merit can then be obtained multiplying its maximal normalized power by its normalized bandwidth.

Using Williams and Yates model, this figure of merit equals a constant, as shown in (1.12).

$$
F o M=P_{\max }^{\prime} \cdot \Delta \Omega=\left\{\begin{array}{lll}
2 & \text { if } & \xi_{H}=\xi_{L} \\
2 \sqrt{2} & \text { if } & \xi_{H}=\xi_{\text {Hopt }}(\Omega)
\end{array}\right.
$$

From this analysis based on Williams and Yates simple model, some general rules for VEHs can be drawn:

- Increasing the intrinsic losses implies that higher damping induced by energy harvesting is required to reach the maximal power. This means that the electromechanical coupling has to be larger for VEH exhibiting large intrinsic losses.

- Lowering the intrinsic losses of the VEH increases the maximal power but decreases the frequency bandwidth. The FoM is however unchanged provided that the optimal energy harvesting damping can be reached.

- The performances can be increased by tuning the damping induced by energy harvesting as a function of the frequency. Higher electromechanical coupling is then required out of the resonance frequency.

Figure 4 (upper plot) shows the normalized maximal power and bandwidth that can be derived from Williams and Yates model as a function of the damping ratio $\xi_{\text {Hmax }} / \xi_{L}$, where $\xi_{\text {Hmax }}$ is the maximal energy harvesting induced damping. It is shown that for $\xi_{H \max }<\xi_{L}$, the normalized power limit $\left(Q_{M}\right)$ cannot be obtained. If $\xi_{\operatorname{Hmax}} \geq \xi_{L}$, the power limit is reached at the resonance frequency and for $\xi_{H}=\xi_{L}$. The normalized bandwidth limit $\left(4 \sqrt{2} \xi_{L}\right)$ is reached for $\xi_{\text {Hmax }} \geq 3 \xi_{L}$. In this case, $\xi_{H}$ has to be set equal to $\xi_{L}$ for $\Omega=1$, and has to be increased for $\Omega \quad$ r lower than 1. $\xi_{H}$ will thus be equal to $3 \xi_{L}$ for $\Omega=\Omega_{1}$ and $\Omega=\Omega_{2}$ (Eq. (1.11)).

In conclusion of this sub-section, Williams and Yates model is very simple but allows understanding the relationship between the maximal power, the bandwidth and the damping induced by both the energy harvesting process and the intrinsic losses. It however exhibits a major limitation: In this model, the electrical load has no effect on the natural frequency of the $\mathrm{VEH}$; it only affects the damping. Yet, in practical VEH, especially in the case of piezoelectric VEH, 
Nonlinear Conditioning Circuits for Piezoelectric Vibration Energy Harvesters

the electrical side of energy harvesting (i.e. the interface circuit) also impacts the resonance frequency. This effect will be further evidenced in the next sections of this chapter.
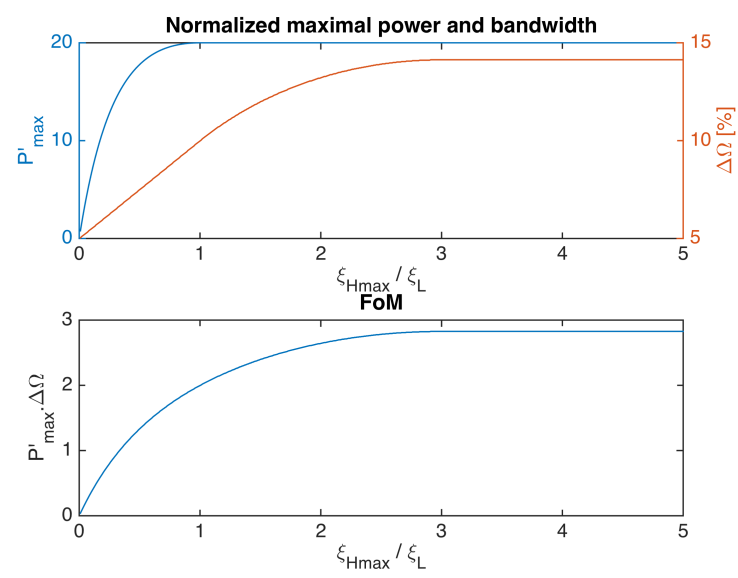

Figure 4. Upper plot: normalized power and bandwidth as a function of the ratio $\xi_{\text {Hmax }} / \xi_{L}$ (for $\xi_{L}=0.025$ ). Lower plot: FoM as a function of the ratio $\xi_{\operatorname{Hax}} / \xi_{L}$ (this plot is independent on $\xi_{L}$ ) 


\section{MODELING AND PARAMETER IDENTIFICATION FOR PIEZOELECTRIC VEH}

\subsection{Model for piezoelectric vibration energy harvester}

Based on electromechanical transduction principles and the electrical and mechanical equations of equilibrium, lumped electromechanical modelling of electromechanical transducers is known as a very effective method [3], [4]. In theory, such models are exact with no restrictions other than linearity, within the limits of the assumptions on boundary conditions and within the frequency range covered by the modeled resonant frequencies.

Most inertial piezoelectric vibration energy harvesters are based on a linear mechanical oscillator (a cantilever beam with a tip mass for instance) including one or several piezoelectric patches.

The very simple PVEH lumped model presented in Figure 5 then provides an accurate and reliable behavioral representation of such PVEH excited around one of their resonant frequencies, as described in [2].

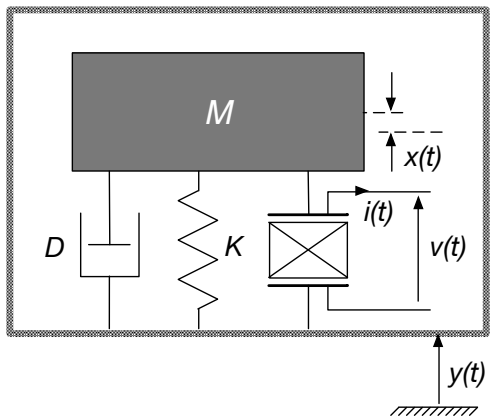

a)

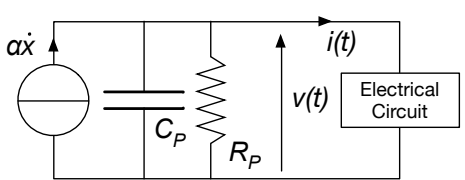

b)

Figure 5. Schematic of a PVEH, a) mechanical point of view, b) electrical point of view

A piezoelectric element represents the piezoelectric transducer(s) (electrically connected together in series or parallel if several are used). It is characterized by its electromechanical force factor $\alpha$, its clamped capacitance $C_{P}$ and its parasitic resistance $R_{P} ; A_{P}=1 / R_{P}$ is the parasitic conductance. $M$ is the equivalent dynamic mass, $D$ is the damping coefficient corresponding to the mechanical losses and $K$ represents the stiffness of the system when the piezoelectric element is short-circuited.

An external vibration $y(t)$ is applied to the system, inducing a relative displacement $x(t)$ between the mass and the housing. As a consequence of the mechanical stress variation, an AC voltage $v$ appears between the piezoelectric electrodes and a current $i$ is generated if an electrical load is connected. Since most applications require a DC voltage, the electrical load usually implements an AC/DC conversion stage.

The governing equations of such an electromechanical system are given by Eqs. (2.1), where $\gamma$ is the ambient acceleration.

$$
\left\{\begin{array}{l}
M \ddot{x}+K x+D \dot{x}+\alpha V=M \gamma \\
I=\alpha \dot{x}-C_{P} \dot{V}-A_{P} V
\end{array}\right.
$$

$\omega_{0}$, the natural frequency of the short-circuited PVEH is given by:

$$
\omega_{0}=\sqrt{\frac{K}{M}}
$$


Three dimensionless parameters are used for the characterization of PVEH: the electromechanical coupling coefficient squared $k^{2}$, the mechanical losses damping ratio $\xi_{M}$ and the resistive losses coefficient $\xi_{E}$, whose expressions are:

$$
k^{2}=\frac{\alpha^{2}}{K C_{0}+\alpha^{2}} \quad \xi_{M}=\frac{D}{2 \sqrt{K M}} \quad \xi_{E}=\frac{1}{2 R_{P} C_{P} \omega_{0}}
$$

$k^{2}$ describes the effectiveness of quasi-static energy conversion between electrical and mechanical forms. For a PVEH in open-circuit subjected to a quasi-static stress, it is equal to the electrostatic energy divided by the total energy in the system. A modified coupling coefficient $k_{m}^{2}$, defined as (2.4), is introduced to simplify theoretical expressions. It is, too, an indicator for the electromechanical coupling, insofar as it represents the quotient between the electrostatic energy and the elastic energy in the generator (also in quasi-static operation). Note that despite $k^{2}$ remains always lower than $1\left(k^{2}=1\right.$ meaning that all the input mechanical energy is converted into electrostatic energy), $k_{m}^{2}$ is not limited. For weakly coupled generators, the values of $k^{2}$ and $k_{m}^{2}$ are close one to the other.

$$
k_{m}^{2}=\frac{\alpha^{2}}{K C_{P}}=\frac{k^{2}}{1-k^{2}}
$$

\section{$2.2 \quad$ Electrical model for SPICE-type simulations}

Using the classical analogies between electrical and mechanical figures (force and voltage; velocity and current), the equivalent electrical network shown in Figure 6 can be derived for the modeling of PVEH. The $\alpha V$ voltage source and the $\alpha \dot{x}$ current sources correspond to the electromechanical transduction. The mechanical power absorbed in the voltage source equals the electrical power provided by the current source, reflecting a lossless energy conversion. The mechanical branch includes an electrical oscillator $\left\{L_{m}, C_{m}, R_{m}\right\}$ that represents the mechanical resonance. The electrical branch is the same as the one depicted in Figure 5b). The governing equations from this electrical network are identical to Eqs. (2.1) provided that $L_{m}=M, C_{m}=K^{-1}$ and $C_{m}=D$.

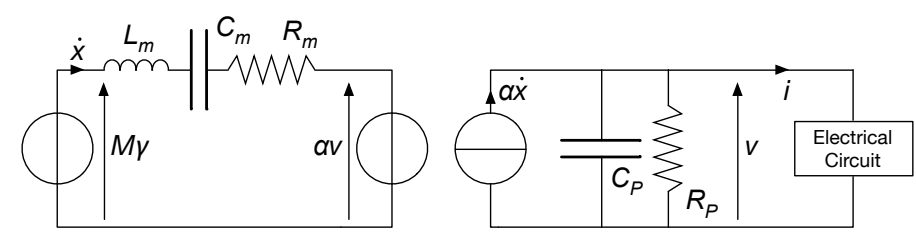

Figure 6. Equivalent electrical network for a PVEH

This model can be easily implemented in SPICE-based software (SPICE: Simulation Program with Integrated Circuit Emphasis) and can then be used for efficient simulation of PVEH with various electrical interface and ambient acceleration profiles.

As an example, Figure 7 is a screenshot of a schematic implemented in the LTSPICE ${ }^{\text {TM }}$ Software (Linear Technologies Corporation), where a classical full bridge rectifier is used as an AC/DC electrical interface between the PVEH and the resistor $R_{L}$, which models the input resistance of the circuit to be powered. 


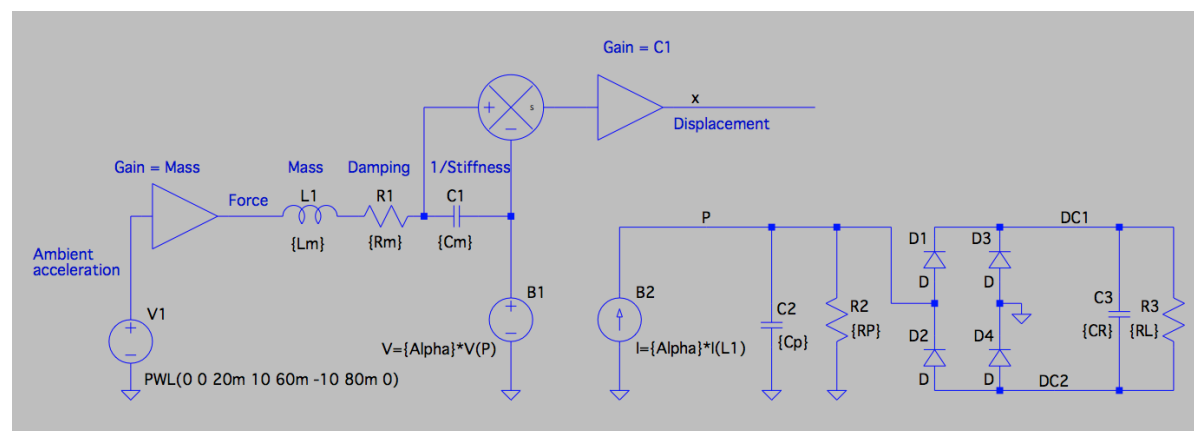

Figure 7. PVEH combined with the classical full bridge rectifier interface (detailed in section 4) modeling using LTSPICE ${ }^{\text {TM }}$ Software.

\subsection{Model identification procedure}

The parameters of the model presented in the previous subsection can be derived from a given PVEH structure using analytical or finite element modeling approaches [5] [6], provided that the characteristics and dimensions of the used materials are known with sufficient accuracy. In practice, the mechanical losses related to mechanical assembly of the different parts of a PVEH are not easy to predict (i.e. bonding, clamping, etc.). The piezoelectric characteristics provided by manufacturers may also exhibit important uncertainty and variability. This explains why significant discrepancies are usually observed between theoretical PVEH characteristics and experimental ones.

This subsection details a very convenient procedure to determine experimentally the actual electromechanical parameters of the model based on a simple measurement of the PVEH complex admittance. The measurement of the complex admittance of the PVEH has to be performed around its natural frequency. This can be done using an impedance analyzer. During this measurement, the PVEH should not be excited by ambient acceleration.

Using equations (2.1) written in the frequency domain, and the dimensionless parameters $k_{m}^{2}, \xi_{E}$ and $\xi_{M}$, the expression of the PVEH admittance can be obtained:

$$
\underline{Y_{P}}=-\frac{\underline{l}}{\underline{V}}=j C_{P} \omega\left(1+\frac{k_{m}^{2}}{1-\Omega^{2}+2 j \xi_{M} \Omega}-\frac{2 j \xi_{E}}{\Omega}\right)
$$

It is shown that the admittance is the one of a $C_{P}$ capacitor multiplied by a dimensionless factor only function of $\Omega, k_{m}^{2}, \xi_{E}$ and $\xi_{M}$. Figure 8 shows the comparison of the experimental and modeled admittance of a real PVEH. The identification of the parameters has been performed using optimization methods to get the best match between the measurements and the model.
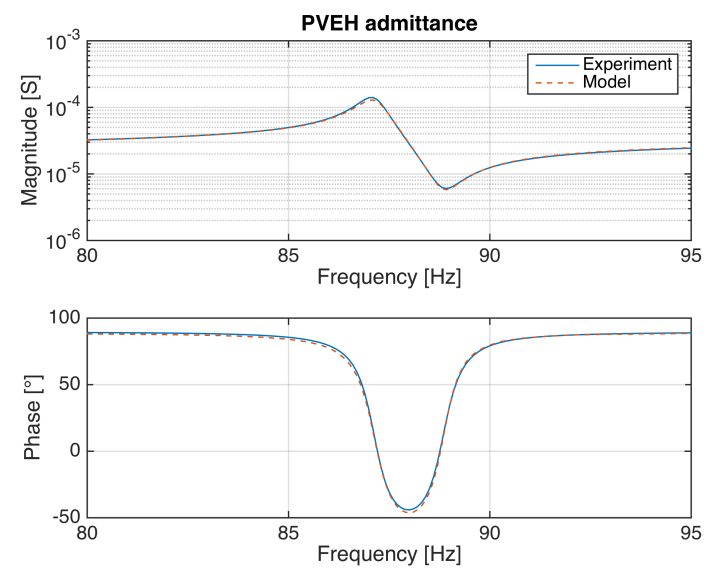

Figure 8. An example of the experimental and modeled admittance of a PVEH 
Five parameters can be obtained from the measurement of the admittance: $C_{P} \omega_{0}, k_{m}^{2}, \xi_{E}$ and $\xi_{M}$. From them, it is not possible to get all the six parameters of the constitutive equations (2.1). To get $M, K, D$ and $\alpha$, an additional measurement is indeed required. For instance, the ratio in the frequency domain of the open-circuit voltage $v_{0}$ to the displacement of the dynamic mass $x$ can be used to determine $\alpha$ as shown by Eq. (2.6). From Eqs (2.2) and (2.3), $M, K$ and $D$ can eventually be calculated.

$$
\frac{v_{0}}{\underline{x}}=\frac{\alpha}{C_{P}} \frac{1}{1-2 j \frac{\xi_{E}}{\Omega}} \approx \frac{\alpha}{C_{P}}
$$

Finally, the six independent identified parameters for the considered PVEH model are listed in Table 1.

Among other identification procedure which can be found in literature, a classical one consists in determining the coupling coefficient $k^{2}$ from the open-circuit and short-circuit resonance frequencies of the PVEH, and the mechanical damping ratio $\xi_{M}$ from the $-3 \mathrm{~dB}$ frequency bandwidth of the displacement $x$ of the PVEH with piezoelement short-circuited as electrical boundary condition [7].

electrical losses coefficient $\xi_{E}$ is usually much lower than 0.01 and its effect on the admittance and more generally on the PVEH performances is weak in most cases. Consequently, $\xi_{E}$ is neglected in most of the literature.

Table 1 PVEH identified parameters

\begin{tabular}{ll|ll}
\hline$C_{0}$ & $51.7 \mathrm{nF}$ & $\xi_{E}$ & $2.8610^{-3}$ \\
$\omega_{0}$ & $547 \mathrm{rad} / \mathrm{s}$ & $\xi_{M}$ & $4.1410^{-3}$ \\
$\boldsymbol{k}_{\boldsymbol{m}}^{2}$ & $3.97 \%$ & $M$ & $10.2 \mathrm{~g}$ \\
\hline
\end{tabular}




\section{OPTIMAL IMPEDANCE MATCHING}

Several works used the well-known optimal impedance matching strategy to maximize the power output of PVEH [5], [8]. In this section the optimal linear electrical load that maximizes the PVEH power generation will be analytically determined. As a first approach, no technical limitation will be taken into account. Results that are not achievable in practice may be obtained, but this study will theoretically define the PVEH performance upper boundary.

\subsection{Theory}

An electrical load modeled by the complex admittance $\underline{Y_{L}}=A_{L}+j B$ is connected to the PVEH, as shown in Figure 9.

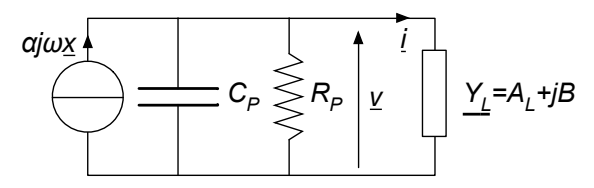

Figure 9. Electrical network using the matching impedance strategy

The piezoelectric voltage $v$ can then be expressed in the frequency domain as:

$$
\underline{v}=\frac{\alpha j \omega \underline{x}}{A_{P}+A_{L}+j\left(C_{P} \omega+B\right)}
$$

which can be rewritten as Eq. (2.8), where $v$ and $v$ are two dimensionless coefficient respectively corresponding to the in-phase and the quadrature-phase components of the voltage with respect to the displacement.

$$
\underline{v}=\frac{\alpha}{C_{P}}(v+j v) \underline{x} \quad \text { with }\left\{\begin{array}{l}
v=\frac{C_{P} \omega\left(B+C_{P} \omega\right)}{\left(A_{P}+A_{C}\right)^{2}+\left(B+C_{P} \omega\right)^{2}} \\
v=\frac{C_{P} \omega\left(A_{P}+A_{C}\right)}{\left(A_{P}+A_{C}\right)^{2}+\left(B+C_{P} \omega\right)^{2}}
\end{array}\right.
$$

Substituting Eq. (2.8) in the mechanical constitutive equation (2.1) written in the frequency domain leads to the expression of the displacement:

$$
\underline{x}=\frac{M \underline{\gamma}}{K-M \omega^{2}+\frac{\alpha^{2}}{C_{P}} v+j\left(C \omega+\frac{\alpha^{2}}{C_{P}} v\right)}
$$

Normalizing the displacement with respect to $\gamma_{m} / \omega_{0}^{2}$ (the ambient displacement magnitude) gives:

$$
\underline{x}^{\prime}=\frac{1}{1-\Omega^{2}+k_{m}^{2} v+j\left(2 \xi_{m} \Omega+k_{m}^{2} v\right)}
$$

This expression shows that the natural frequency of the PVEH can be tuned through $v$ and that the damping induced by energy harvesting can be tuned through $v(v>0)$. The larger $k_{m}^{2}$, the larger the effect of $v$ and $v$ variations.

The harvested energy is considered to be the energy dissipated in the real component of the electrical load $\left(A_{L}\right)$. Of course, this does not correspond to a realistic energy harvesting circuit since the voltage would need to be rectified. $A_{L}$ can however be considered as the equivalent 
input conductance of the circuit to be supplied. This approach aims at providing an upper limit to the performance of a PVEH.

In these conditions, the harvested power can be expressed as:

$$
P=\frac{|\underline{v}|^{2}}{2} A_{L}
$$

In the case where $\xi_{E}$ is neglected $\left(A_{P}=0\right)$, intrinsic losses in the PVEH are modeled by $\xi_{M}$ only. It follows from Williams and Yates analysis and from Eq. (2.10) that the power is maximized when $v$ is chosen so that the natural frequency of the PVEH matches the operation frequency and $v$ so that the damping induces by the energy harvesting equals the intrinsic damping, which gives:

$$
\left\{\begin{array}{l}
v=\frac{\Omega^{2}-1}{k_{m}^{2}} \\
v=\frac{2 \xi_{M} \Omega}{k_{m}^{2}}
\end{array}\right.
$$

If no technical constraint is taken into account, $v$ can take any positive or negative value and $v$ can take any positive value, which means that Eqs (2.12) can be verified whatever the value of $\Omega$. In this case, the power limit $P_{\text {lim }}$ given by Eq. (1.5) is obtained, whatever the operation frequency.

If $\xi_{E}$ is not neglected $\left(A_{P} \neq 0\right)$, the optimal values of $A_{L}$ and $B$ that maximize the harvested power are obtained as a function of $\Omega$ by looking for the roots of the derivatives of the harvested power with respect to $A_{L}$ and $B$ respectively. They are given by:

$$
\left\{\begin{array}{l}
A_{\text {Lopt }}=C_{P} \omega\left(\frac{2 \xi_{E}}{\Omega}+\frac{2 k_{m}^{2} \xi_{m} \Omega}{4 \Omega^{2} \xi_{m}^{2}+\left(\Omega^{2}-1\right)^{2}}\right) \\
B_{o p t}=C_{P} \omega\left(\frac{k_{m}^{2}\left(\Omega^{2}-1\right)}{4 \Omega^{2} \xi_{m}^{2}+\left(\Omega^{2}-1\right)^{2}}-1\right)
\end{array}\right.
$$

And the maximal power that can be harvested at a given operation frequency by:

$$
P_{\text {opt }}=\left.P\right|_{A_{L}=A_{\text {Lot }} \text { and } B=B_{\text {oot }}}=\frac{M \gamma_{M}^{2}}{16 \omega_{0}} \frac{k_{m}^{2} \Omega^{2}}{k_{m}^{2} \xi_{M} \Omega^{2}+\xi_{E}\left(4 \xi_{M}^{2} \Omega^{2}+\left(\Omega^{2}-1\right)^{2}\right)}
$$

The normalized optimal power is then:

$$
P_{o p t}^{\prime}=\frac{1}{2 \xi_{M}+\frac{2 \xi_{E}}{k_{m}^{2}}\left(4 \xi_{M}^{2}+\frac{\left(\Omega^{2}-1\right)^{2}}{\Omega^{2}}\right)}
$$

As previously mentioned if $\xi_{E}=0$, the optimal power reaches $P_{\text {lim }}$ whatever the operation frequency, provided that $A_{L}$ and $B$ are adequately tuned. If $\xi_{E}$ is not neglected, it is shown that the optimal power reached a maximum for $\Omega=1$. Equation (2.16) gives the normalized maximal power.

$$
P_{\max }^{\prime}=\frac{1}{2 \xi_{M}\left(1+4 \frac{\xi_{E} \xi_{M}}{k_{m}^{2}}\right)}
$$


The PVEH bandwidth can be obtained finding $\Omega_{1}$ and $\Omega_{2}$ so that $P_{\text {opt }}^{\prime}\left(\Omega_{1}\right)=P_{\text {opt }}^{\prime}\left(\Omega_{2}\right)=P_{\max }^{\prime} / 2$. The normalized bandwidth is then given by (2.17), and the bandwidth by $\Delta \omega=\Delta \Omega \cdot \omega_{0}$.

$$
\Delta \Omega=\Omega_{2}-\Omega_{1}=2 \xi_{M} \sqrt{1+\frac{k_{m}^{2}}{4 \xi_{E} \xi_{M}}}
$$

The normalized power and displacement are plotted in Figure 10 as a function of the operating frequency for different values of $\xi_{E}$. The corresponding real and complex parts of the optimal electrical load admittance are shown in Figure 11. It is clearly shown that $\xi_{E}$ drastically affects the bandwidth of the PVEH. From Figure 11, it can be seen that the optimal complex part of the load admittance is independent on $\xi_{E}$ and that its sign is mainly negative in the frequency range but can also be positive. This concretely means that the optimal load is a resistor in parallel with an inductor in most of the frequency range but that it is sometimes a resistor in parallel with a capacitor (for operation frequency slightly above the natural frequency of the PVEH).
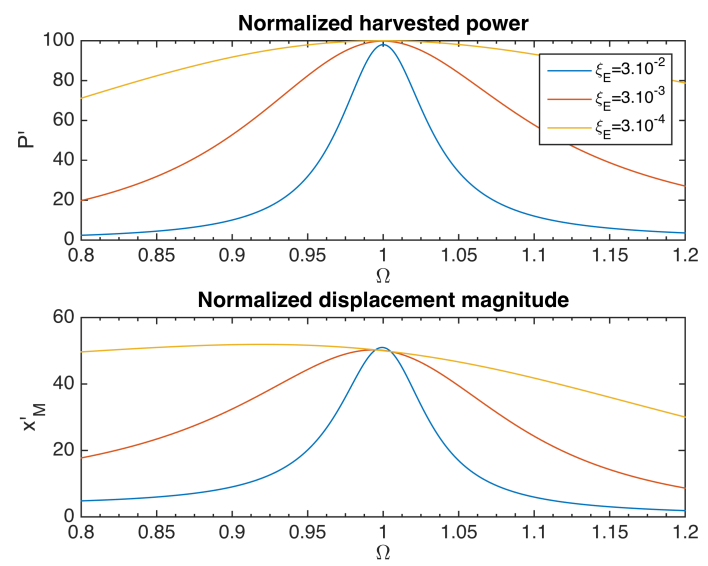

Figure 10. Normalized harvested power (upper plot) and displacement magnitude (lower plot) as a function of the operation frequency for different values of $\xi_{E}\left(k_{m}^{2}=3 \%, \xi_{M}=0.005\right)$
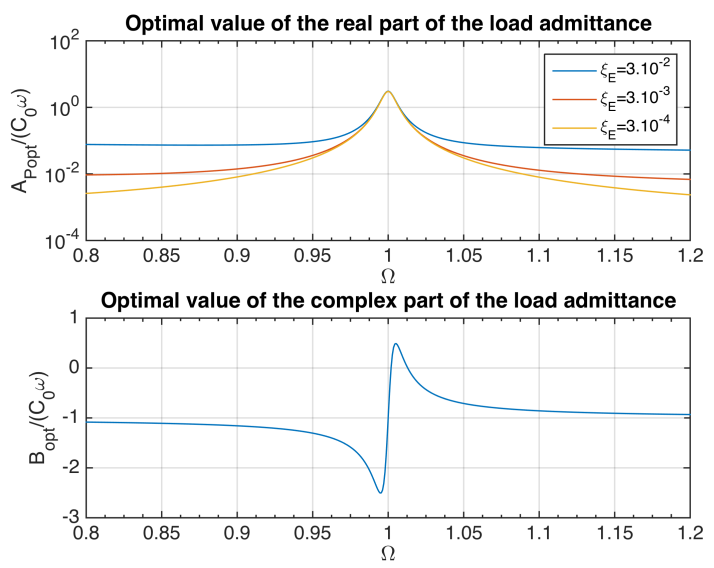

Figure 11. Real (upper plot) and complex (lower plot) parts of the optimal electrical load admittance as a function of the operation frequency for different values of $\xi_{E}\left(k_{m}^{2}=3 \%, \xi_{M}=0.005\right)$

Figure 12 exhibits the voltage on the piezoelectric element when the optimal complex electrical load is selected as a function of the operating frequency. It is clearly shown that increasing the bandwidth lead to very high piezoelectric voltage when the operating frequency is shifted away from the resonance frequency (up to six times the maximal open-circuit voltage in the 
considered cases). This is due to the increase of reactive power alternatively flowing between the capacitance of the piezoelectric element and the complex part of the optimal admittance.

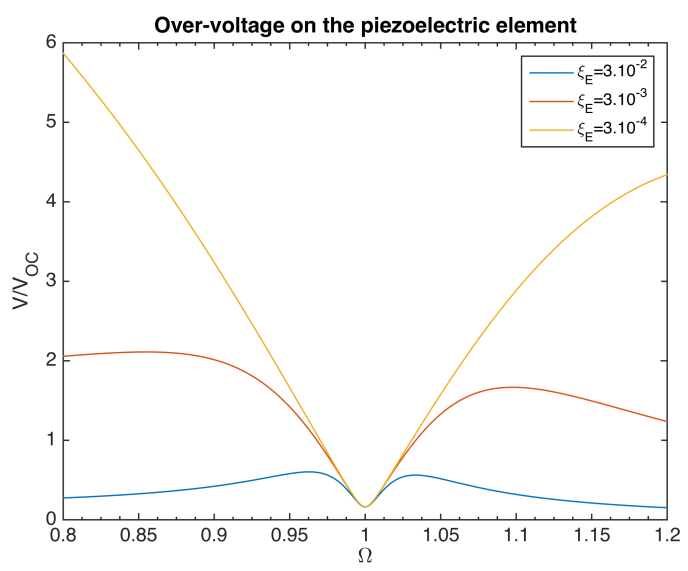

Figure 12. Ratio of the optimal piezoelectric voltage to the maximal open-circuit voltage as a function of the operation frequency for different values of $\xi_{E}\left(k_{m}^{2}=3 \%, \xi_{M}=0.005\right)$

Finally the figure of merit that is equals to the product of the maximal normalized power by the normalized product is given by:

$$
\text { FoM }=P_{\max }^{\prime} \cdot \Delta \Omega=\frac{\frac{k_{m}^{2}}{4 \xi_{E} \xi_{M}}}{\sqrt{1+\frac{k_{m}^{2}}{4 \xi_{E} \xi_{M}}}}=\frac{\chi}{\sqrt{1+\chi}} \text { with } \chi=\frac{k_{m}^{2}}{4 \xi_{E} \xi_{M}}=\frac{\alpha^{2} R_{P}}{D}
$$

Where $\chi$ is dimensionless parameter that can be seen as an indicator of the performance of the PVEH (it increases with the coupling coefficient and decreases with the electrical and mechanical losses).

The normalized power and bandwidth, as well as the FoM are plotted in Figure 13 as a function of $\chi \quad$ an be seen that the FoM can largely exceed $2 \sqrt{2}$, which was the upper limit obtained from the Williams and Yates model. This is because of the frequency tuning mechanism induced by the complex part of the load admittance. For typical PVEH, the order of magnitude for $k_{m}^{2}$ is around $10^{-2}$, whereas it is between $10^{-2}$ and $10^{-3} \quad \xi_{E} \quad \xi_{M}$. This means that $\chi$ typical values ranges from 10 to 100 about. In this case, the figure of merit can be approximated by $F O M \approx \sqrt{\chi}$.

In practices, this impedance matching strategy can hardly be implemented because it requires the realization of complex electrical load with tuning mechanisms. One could think about using active gyrator circuits to emulate large tunable inductors. Such synthetic inductors based on operational amplifiers, capacitances and resistances however consume a lot of power and are not a viable option for energy harvesting applications.

Another limitation of this approach can be inferred from Figure 12: the voltage increase due to the reactive part of the complex optimal load may lead to very high piezoelectric voltage for which depoling effect or at least nonlinearities in the piezoelectric coefficients may be observed.

Possible practical solution for implementing this impedance matching strategy will be discussed in the next section 

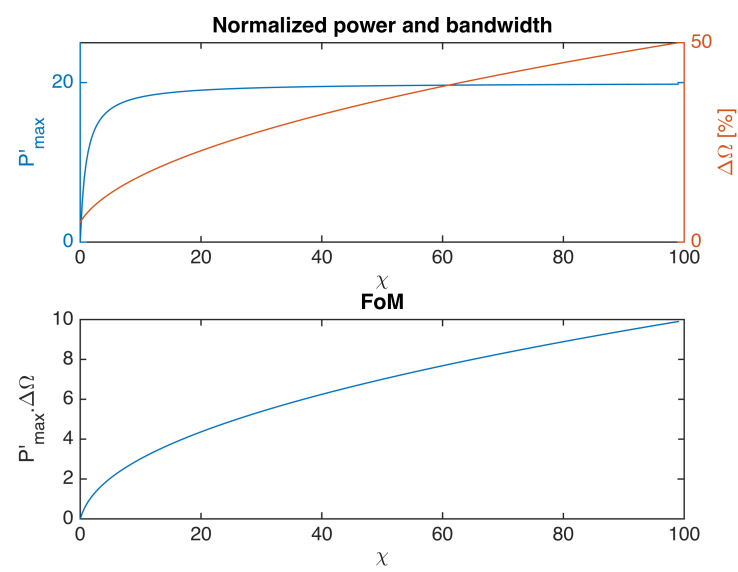

Figure 13. Upper plot: normalized power and bandwidth as a function of $\chi$ (for $\xi_{L}=0.025$ ). Lower plot: FoM as a function of $\chi$ (this plot is independent on $\xi_{L}$ )

\subsection{Practical implementation}

Modern power electronics offers many solutions based on switching-mode converters enabling high-efficiency electrical power conversion. Input and output voltages can exhibit various shapes and polarities, and the power transfer can be unidirectional or bi-directionnal, depending on the considered circuits [9].

In practice, electric loads powered by energy harvesters -such as wireless sensor nodes for instance- don't behave at all like the "optimal matching impedance" defined in the previous subsection. Indeed, such electric loads require DC voltage-regulated power supply, and their consumption may be extremely variable in time. Thus, AC-DC power conversion, energy storage and voltage regulation are the minimum requirements for the electronic interface, which will be used to transfer the electrical energy produced by the piezoelectric material to the electric load.

Passive AC-DC converters, such as diode rectifiers, are very simple to implement but the shape of their AC input voltage and current is not similar to that of linear impedances. Emulation of linear impedance is however possible using PWM rectifiers. The proposed implementation of the optimal impedance matching strategy is based on the interface circuit represented in Figure 14, which is presented in [10]. In this circuit, the AC input of the PWM rectifier is connected to the piezoelectric element. Through the PWM control of the switches of the active rectifier, it is theoretically possible to emulate any linear or nonlinear load, including the optimal impedance defined previously. The DC output of the active rectifier is connected to and energy storage element (a capacitor or a supercapacitor) whose voltage may vary, depending on the energy stored. The output DC voltage delivered to the electric load can be then regulated using a buckboost converter. This sub-circuit enables to ensure output voltage regulation whether the output DC voltage $V_{L}$ is lower or higher than the input DC voltage $V_{D C}$.

The control strategy of this interface circuit is not very complicated, but it is out of the scope of this chapter and it will not be detailed here. Among important ideas to have in mind, practical implementation of the optimal impedance matching strategy is possible. However, in case of very low harvested power level, typically in the range of a few tenths of microwatts or below, the available power may be too low to implement sophisticated control circuits. Therefore, "less optimal" approaches based on simpler control principles and simpler circuits may turn out to be much more efficient in practice. This is the objective of the various techniques developed in the next sections. 
Nonlinear Conditioning Circuits for Piezoelectric Vibration Energy Harvesters

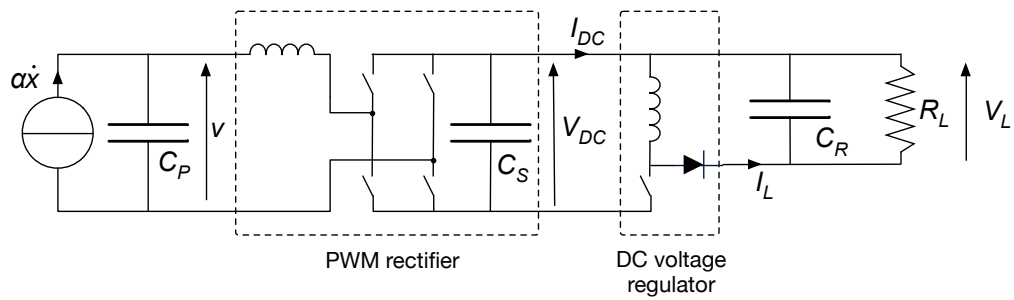

Figure 14. Interface circuit for practical implementation of the optimal impedance matching strategy 


\section{THE CLASSICAL RECTIFIER FOLLOWED BY A RESISTIVE LOAD}

In this section the classical rectifier circuit followed by a storage/smoothing capacitor is studied. This circuit is shown in Figure 15, where $R_{L}$ represents the equivalent input resistance of the device to be supplied. The harvested power is then calculated as the power dissipated into $R_{L}$.

For simplicity, it will be assumed that the electrical losses can be neglected $\left(\xi_{E}=0\right)$. This assumption is valid in most of practical cases where $R_{L}$ is much lower than $R_{P}$.

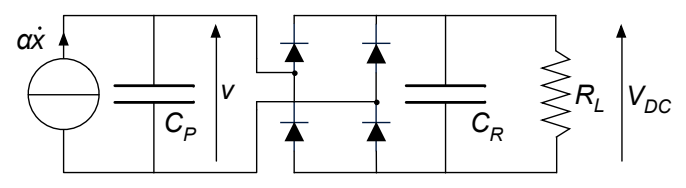

Figure 15. Electrical network using the classical rectifier circuit

\subsection{Power and Bandwidth}

This circuit has been studied in details by Shu and Lien in [11]. Sinusoidal ambient accelerations around the resonance frequency of the PVEH are considered, and it is assumed that the smoothing capacitor is large enough so that $V_{D C}$ is ripple free. The value of $V_{D C}$ can then be obtained as a function of the magnitude $x_{M}$ of the dynamic mass relative displacement:

$$
V_{D C}=\frac{\alpha \omega R_{L}}{C_{P} \omega R_{L}+\frac{\pi}{2}} x_{M}
$$

Because of the nonlinear behavior of the full-wave rectifier, the piezoelectric voltage $v$ is periodic but not sinusoidal. However, since only its fundamental harmonic frequency is close from the resonance frequency of the $\mathrm{VEH}$, it can be assumed that only this fundamental component $v_{1}$, given in the frequency domain by (3.2), impacts the displacement of the VEH dynamic mass.

$$
\underline{v}_{1}=\frac{\alpha}{C_{P}}(v+j v) \underline{x} \text { with }\left\{\begin{array}{c}
v=\frac{\omega R_{L} C_{P}}{\omega R_{L} C_{P}+\frac{\pi}{2}} \\
v=\frac{2 \omega R_{L} C_{P}}{\left(\omega R_{L} C_{P}+\frac{\pi}{2}\right)^{2}}
\end{array}\right.
$$

The expression in the frequency domain of the displacement and the normalized displacement are then the same as in section 3.1, equations (2.9) and (2.10) respectively, except that the expressions of $v$ and $v$ are different.

$$
r^{\prime}=R_{L} C_{P} \omega_{0}
$$

Let $r$ be the normalized load as given by (3.3), $v$ and $v$ can also be expressed as:

$$
\left\{\begin{array}{c}
v=\frac{r^{\prime} \Omega}{r^{\prime} \Omega+\frac{\pi}{2}} \\
v=\frac{2 r^{\prime} \Omega}{\left(r^{\prime} \Omega+\frac{\pi}{2}\right)^{2}}
\end{array}\right.
$$


It can be seen from (3.4) that $v$ is between 0 (when $r^{\prime}=0$ ) and 1 (when $r^{\prime}$ tends to infinite). From (2.10), this means that the resonance frequency of the PVEH is between $\omega_{0}$ (short-circuit resonance frequency) and $\omega_{0} \sqrt{1+k_{m}^{2}}$ (open-circuit resonance frequency).

It can also be seen that $v$ is between 0 (when $r^{\prime}=0$ or when $r^{\prime}$ tends to infinite) and $1 / \pi$ (when $r^{\prime} \Omega=\pi / 2$. From equation (2.12), this means that the optimal damping cannot be obtained if $k_{m}^{2}<2 \Omega \xi_{M} \pi$.

The harvested power can be expressed as:

$$
P=\frac{V_{D C}^{2}}{R_{L}}
$$

And the normalized harvested power is finally given by:

$$
P^{\prime}=8 k_{m}^{2} \frac{r^{\prime} \Omega^{2}}{\left(r^{\prime} \Omega+\frac{\pi}{2}\right)^{2}}\left|\underline{x}^{\prime}\right|^{2}
$$

The maximal normalized power and the corresponding optimal normalized load are plotted as a function of the frequency in Figure 16, for different values of $k_{m}^{2}$. When $k_{m}^{2}$ increases, it can be seen that whereas the bandwidth keeps on increasing, the maximal normalized power first increases and then saturates at $Q_{M}$. It is worthy of note that a continuous tuning of the electrical load as a function of the operation frequency is required to get the best performance. The colored areas represent the load domains for which at least $50 \%$ of the maximal power is harvested.
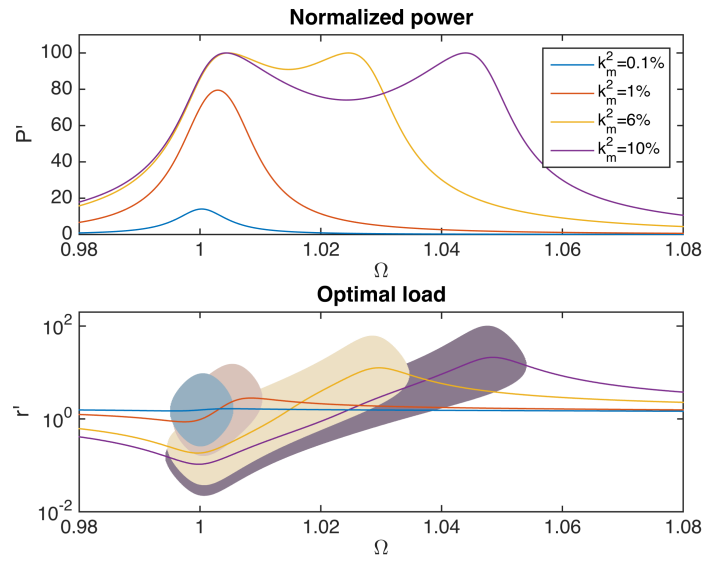

Figure 16. Upper plot: normalized power as a function of $\Omega$ for $\xi_{L}=0.005\left(Q_{M}=100\right)$ and different values of $k_{m}^{2}$. Lower plot: normalized optimal load as a function of $\Omega$ (areas corresponds to the load domain for which the power is at least $50 \%$ of the maximal power)

The normalized power, bandwidth and the figure of merit defined in (1.12) are plotted in Figure 17 as a function of $k_{m}^{2}$ for different values of $Q_{M}$. The black curve in Figure 17a corresponds to $k_{m}^{2} Q_{M}=\pi$. It is shown that the normalized power equals $Q_{M}$ for $k_{m}^{2} Q_{M}>\pi$. Figure $17 \mathrm{~b}$ shows that the bandwidth is quasi linearly increasing with $k_{m}^{2}$. For very large values of $k_{m}^{2}$, the bandwidth however decreases because the power in the well between the two maxima (cf. Figure 16 with $k_{m}^{2}>6 \%$ ) becomes less than half of the maximal power.

For typical PVEH, $k_{m}^{2}$ is in the order of a few percent and $Q_{M}$ is between 10 and a few hundreds. The figure of merit is then usually lower than 5. Figure 17c clearly shows that PVEH with high $k_{m}^{2}$ and high $Q_{M}$ exhibit higher performance. 
It is worthy of note that results presented in Figure 17 imply a continuous tuning of the electrical load as a function of the operation frequency, which requires specific power conversion interface and dedicated MPPT control circuit for practical implementation.

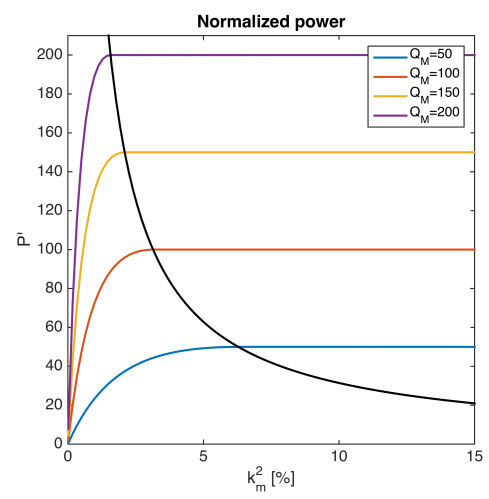

a

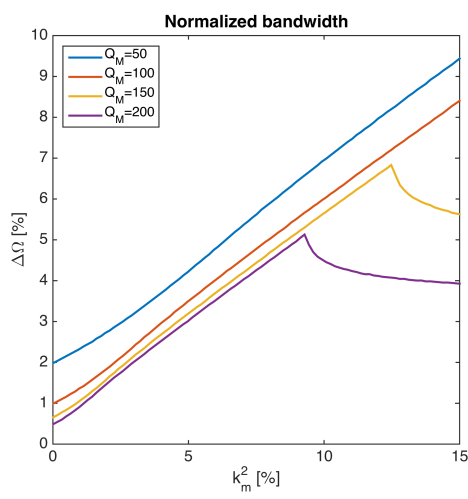

b

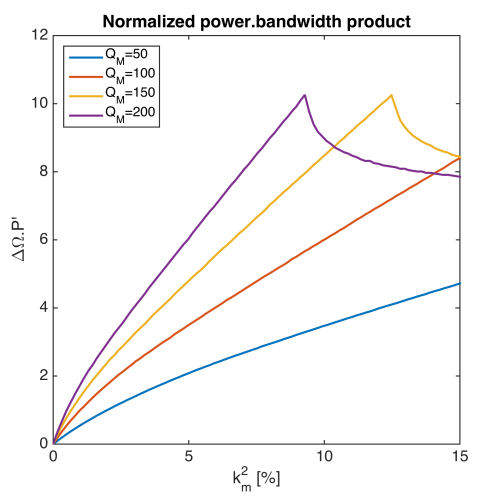

C

Figure 17. a) Normalized power versus $k_{m}^{2}$, b) normalized bandwidth versus $k_{m}^{2}$, c) FoM versus $k_{m}^{2}$

\subsection{Practical implementation}

Several interface circuit based on the classical rectifier circuit principle have been proposed for maximizing the power transfer from the piezoelectric device to the load. In this optimization approach, the main function of the interface circuit is to emulate the optimal load resistance defined in the previous section. Ottman et al studied one of the first interface circuits [12]. This circuit was based on the buck DC-DC converter represented in Figure 18. Using the appropriate control law of the DC-DC converter, it was shown that the performances of the system could be greatly improved. In order to reduce the power consumption of the control circuit so that it can be self-powered, the authors proposed a new control principle of the buck converter that exhibited similar performances, with much simpler implementation [13].

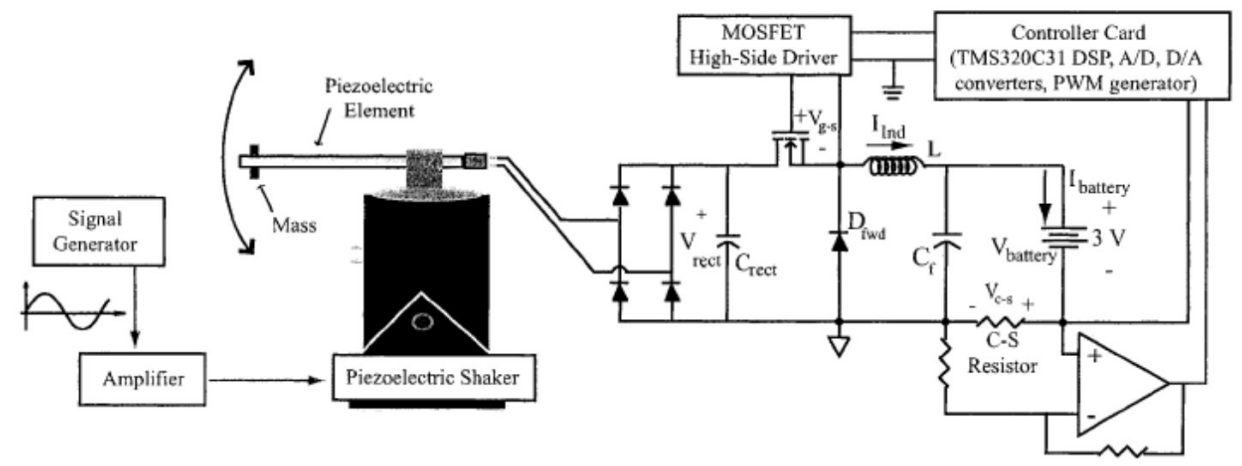

Figure 18. Experimental setup including a buck DC-DC converter (from [12])

Following the same approach, Lefeuvre et al. [14] proposed an interface circuit based on a buckboost DC-DC converter depicted in Figure 19. In discontinuous current mode, this circuit exhibits a constant input resistance for a given duty-cycle control of the electronic switch, making possible the implementation of the interface using only an oscillator with constant dutycycle as control circuit (IC1 in Figure 19). For output powers ranging from $200 \mu \mathrm{W}$ to $1.5 \mathrm{~mW}$, experimental results showed more than $70 \%$ overall efficiency of this circuit, including the control circuit consumption. In this practical example, the input resistance of the interface circuit was predetermined to be as close as possible to the optimal value at the resonant 
frequency. Consequently, the robustness was relatively weak with respect to variations of electromechanical characteristics.

To overcome this drawback, Maximum Power Point Tracking (MPPT) circuits with ultra-low power consumption were implemented. Yi et al. proposed a low-power interface circuit, based on a switched capacitor DC-DC converter, integrated in a $0.35 \mu \mathrm{m}$ CMOS process ASIC [15]. In this ASIC, an energy-adaptive MPPT allowed to activate different operation modes according to the available power. Kong et al. presented an interface circuit based on a flyback DC-DC converter [16]. The MPPT algorithm was implemented using a low-power microcontroller unit MSP430 from Texas Instruments. Experimental results indicated that the proposed interface circuit achieved $72 \%$ efficiency around the resonant frequency and around $8.4 \mathrm{~mW}$ output power.

In this domain, the current trends are clearly to improve the efficiency of low-power DC-DC converters and to design fast and effective MPPT control circuits with ultra-low power consumption [17], [18].
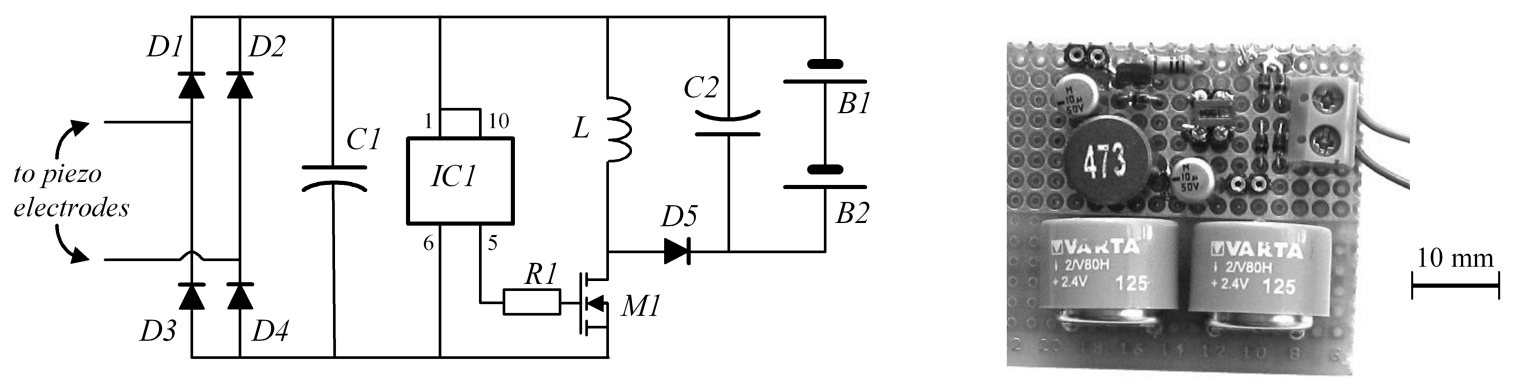

Figure 19. Experimental circuit and setup including a buck-boost DC-DC converter (from [14]) 


\section{NONLINEAR ENERGY HARVESTING CIRCUITS}

\subsection{Principle}

Nonlinear energy extraction approaches have been developed to optimize the energy extracted from PVEH. From the simple model presented in section 2.1, the energy equation (4.1) is obtained multiplying both terms of the dynamical mechanical equilibrium equation by the velocity and integrating over the time variable. It shows that the energy provided by the ambient acceleration is divided into kinetic energy, potential elastic energy, mechanical losses and the energy extracted from the piezoelectric element. Nonlinear energy extraction circuits aim at increasing this last term.

$$
\int M \gamma \dot{x} d t=\frac{1}{2} M \dot{x}^{2}+\frac{1}{2} K x^{2}+\int D \dot{x}^{2} d t+\int \alpha V \dot{x} d t
$$

The basic electronic element for nonlinear energy extraction circuit is shown in Figure 20a. It consists in connecting a coil in series with an electronic switch in parallel to the piezoelectric element. The switch is almost always open, except when a minimum or maximum of the dynamic mass displacement occurs. At this moment, the switch is closed and $C_{P}$ in parallel with $L$ forms an electrical oscillating circuit. The switch is kept closed during half of the oscillating period $T_{I}$, so that the voltage is reversed. Because there are some electrical losses in the $\left\{L, C_{P}\right\}$ network, characterized by the quality factor $Q_{I}$, the absolute value of the voltage after inversion is slightly reduced compared to the one before the switch is closed. Corresponding waveforms for the voltage, displacement and velocity of the dynamic mass are shown in Figure 20b.

This simple circuit has two effects: First, for constant displacement amplitude, the amplitude of the voltage is largely increased; second voltage and velocity are of the same sign. These two effects clearly induce the increase of the energy extraction term of equation (4.1).

This very simple circuit alone is however not suitable for energy harvesting purpose, since the extracted power is not converted into useful power: It is actually dissipated as heat in the coil and the piezoelectric element. This circuit was in fact initially developed for vibration damping purpose and called SSDI (Synchronized Switch Damping on Inductor) [19].

Adding AC/DC and energy storage stages to this elementary circuit has been the basis of the further developments of nonlinear energy harvesting approaches. For instance, the SSHI (Synchronized Switch Harvesting on Inductor) simply consists in combining this switch-coil circuit to the classical full-wave rectifier approach, connecting them in parallel to the piezoelectric element [7].

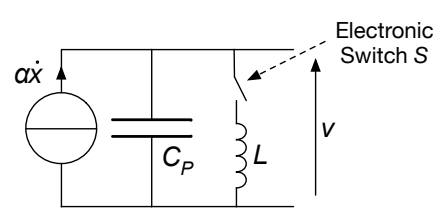

a

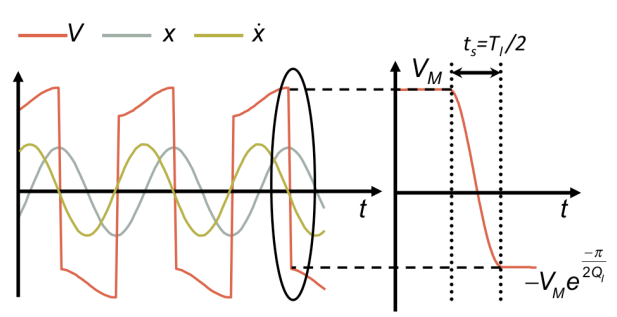

b

Figure 20. a) Basic electronic element for nonlinear energy extraction b) Corresponding typical waveforms.

\subsection{Comparison of various nonlinear circuits}

Based on the general principle exposed previously, several nonlinear energy harvesting circuits with different features have been proposed over the last decade. This subsection outlines the 
main properties of each circuit. They have in common to increase the last term of Eq. (4.1), that is to say they increase the mechanical damping induced by energy conversion. Low-coupling PVEH particularly benefit from these circuits. Indeed, such increase of the electromechanical energy conversion effectiveness allows getting closer to the optimal damping (see section 1.1). This beneficial effect also increases the PVEH performances in case of out-of resonance and pulsed excitation [20]. In the case of strongly coupled PVEH excited at resonant frequency, no gain can be expected if optimal damping is already attained. Specific features of the main nonlinear circuits are detailed hereafter.

The parallel SSHI circuit depicted on Figure 21 is one of the first nonlinear PVEH interfaces based on the principle of "synchronized switching" [21], [7], [20]. This circuit is a straightforward association of the classical rectifier circuit of Figure 15 and the circuit of Figure 20a. Compared to the classical rectifier circuit, the parallel SSHI circuit tends to increase the piezoelectric voltage. The optimal load resistance tends also to be higher than with the classical rectifier circuit. This voltage magnification property may be used to get high voltages, or to reduce the energy losses related to the voltage drop of the diodes in the rectifier bridge. This is particularly interesting in the case of low-voltage PVEH microsystems, whose open-circuit voltage is typically lower than $1 \mathrm{~V}$. Shu et al. studied in detail the effect of this circuit on PVEH as a function of the excitation frequency [22].

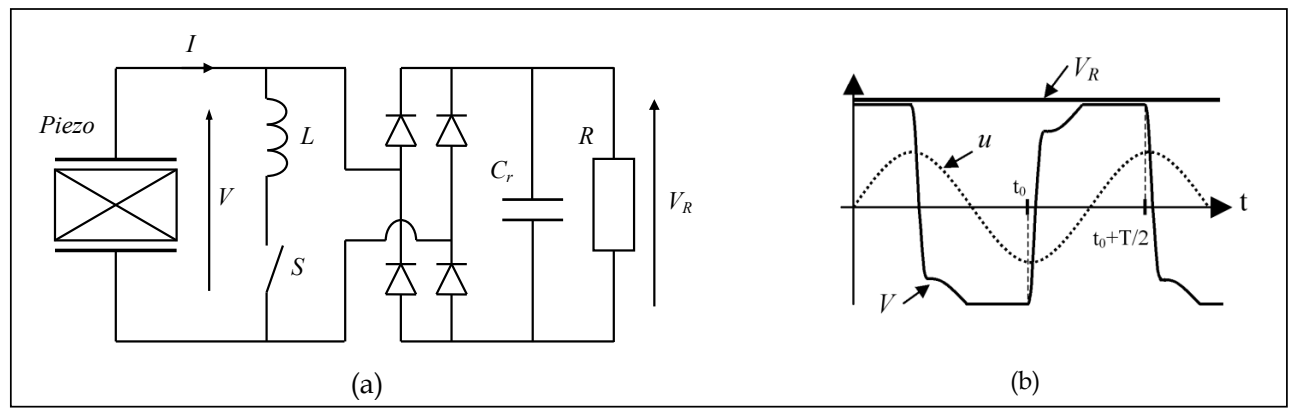

Figure 21. a) Parallel SSHI circuit schematic and b) typical waveforms (from [23])

In case of the series SSHI circuit (Figure 22), the coil-switch dipole is connected in series with the PVEH instead of being connected in parallel. This induces slight changes on the piezoelectric voltage waveform, and significant differences on the circuit output voltage and the optimal load resistance. Indeed, in this case the output voltage and the optimal load resistance are smaller than those of the classical rectifier circuit [23]. Therefore, the series SSHI circuit is particularly interesting to get an output voltage lower than the piezoelectric voltage. This circuit was first proposed for this voltage reduction property by Taylor et al. [24].

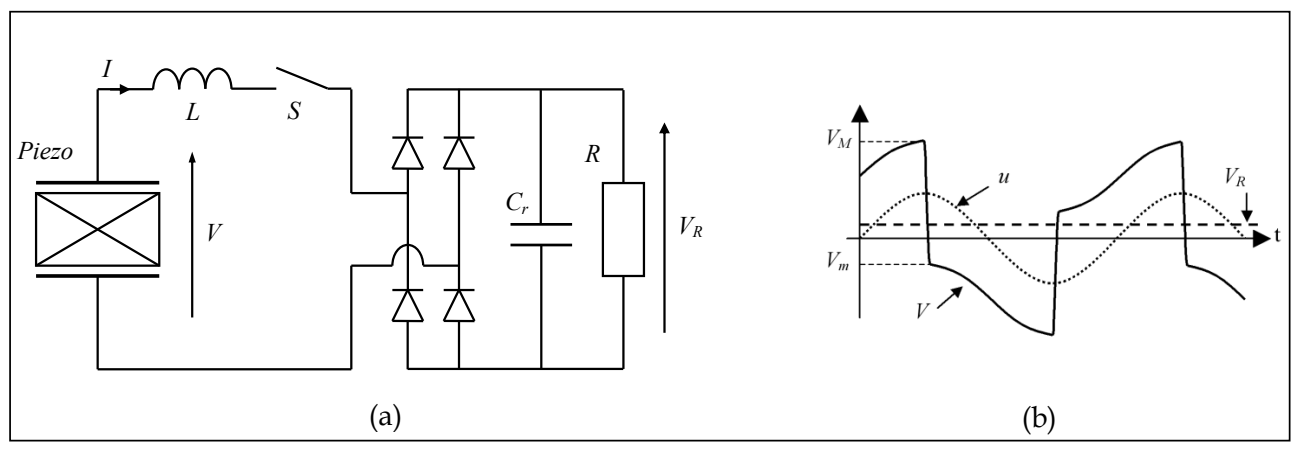

Figure 22. a) Series SSHI circuit schematic and b) typical waveforms (from [23])

The principle of the SECE circuit consists in extracting promptly and entirely the electric energy converted by the piezoelectric element on each extremum of the piezoelectric voltage. In the 
SECE circuit represented on Figure 23, the energy transfer is achieved by a flyback-type DC-DC power converter [25]. Alternatively, the flyback circuit can be replaced by a buck-boost DC-DC converter [26]. In theory, the harvested power is independent of the load. This unique property enables to harvest the maximum power without MPPT system.

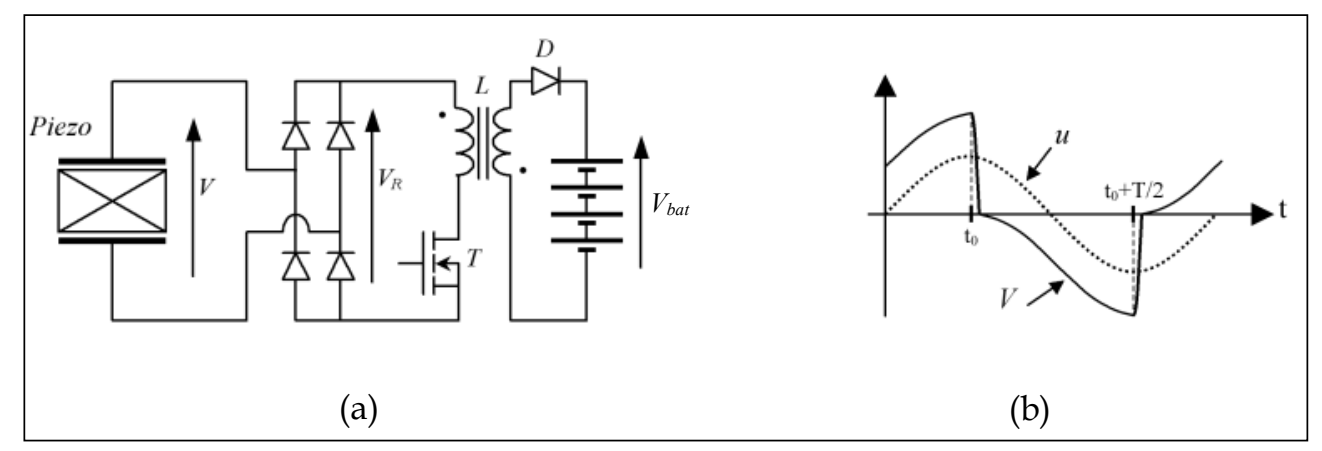

Figure 23. a) SECE circuit schematic and b) typical waveforms (from [25])

Several works focused on efficiency improvement of the previous circuits for low-voltage applications. A way for reducing the voltage drops consisted in reducing the number of diodes used for voltage rectification. In this domain, Makihara et al. [27] proposed a half-bridge circuit for the Parallel SSHI circuit. Lallart et al. proposed another half-bridge circuit for low-voltage implementation of the series SSHI interface [28]

The SSHI-MR circuit proposed by Garbuio et al. [29] (Figure 24) brought an ultimate reduction of the losses due to the threshold voltage of the diodes. The typical waveforms of this circuit are very similar to those of the series SSHI circuit. The main difference comes from the magnetic transformer, which replaces the coil of the series SSHI circuit. The voltage gain of the transformer in association with the single-diode rectifier enables operation towards ultra-lowvoltage PVEH: experimental results showed effective energy harvesting from piezoelectric voltages as low as $30 \mathrm{mV}$.

The single-supply pre-biasing circuit presented by Elliott et al. [30] has piezoelectric waveforms identical to that of the parallel SSHI circuit. The use of MOSFET electronic switches instead of diodes enables efficient operation even with ultra-low PVEH voltages.

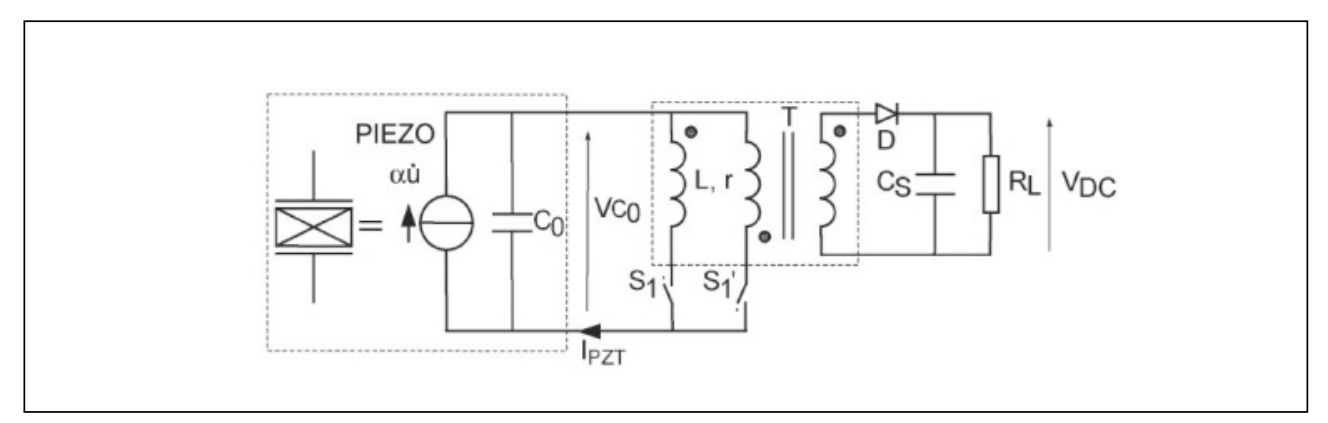

Figure 24. SSHI-MR circuit schematic (from [29]).

The DSSH circuit proposed by Lallart et al. is an association of the Series SSHI circuit and the buck-boost DC-DC converter of the SECE circuit [31] (Figure 25). The intermediate energy storage capacitor $C_{\text {int }}$ brings an additional degree of freedom to control the energy conversion. This intermediate energy tank is used here for optimizing the trade-off between energy harvesting and mechanical damping. In addition, the buck-boost DC-DC converter makes the harvested power optimal whatever the load characteristics (i.e. no influence of the load 
equivalent resistance). The DSSH circuit implementation is a little bit more complicated than that of the SECE or the SSHI circuits. Despites the cumulated losses of the two conversion stages, experimental results exhibited much better performances than that of the standard and SECE techniques in the case of PVEH with small $\mathrm{k}^{2} \mathrm{Q}_{\mathrm{m}}$. The so-called ESSH circuit proposed by Shen et al. [32] can be considered as an improvement of the DSSH circuit, which allows a finer control of the mechanical damping, induced by energy conversion.

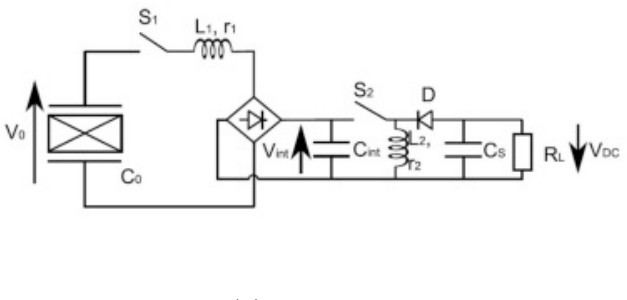

(a)

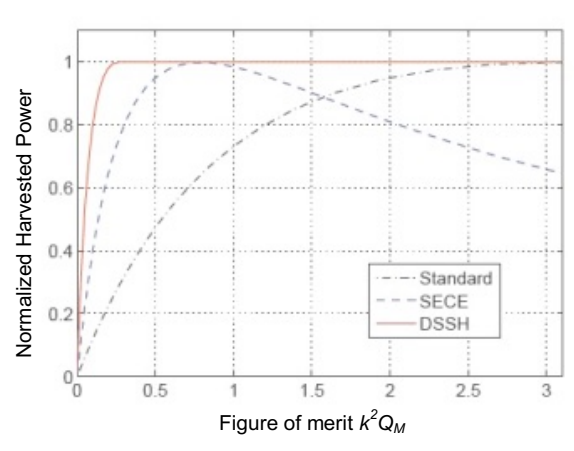

(b)

Figure 25. a) DSSH circuit schematic, and b) power vs. $\mathrm{k}^{2} \mathrm{Q}_{\mathrm{m}}$ at resonant frequency (from [31]).

Based on this general nonlinear approach, several other interface circuits have been proposed. Wu et al. proposed the so-called SSDCI circuit, based on a circuit similar to the series SSHI, but with a modified switch control [33]. The principle of the method consists of transferring the electrostatic energy available on the piezoelectric element to a storage capacitor through an inductance.

Dicken et al. proposed the so-called Pre-Biasing circuit [34], which enables to pre-bias the piezoelectric element with the appropriate voltage to finely optimize the harvested power. However, the complexity of the circuit, which includes numerous switches and two different power supplies, may be an obstacle to low-power standalone implementation.

Energy conversion cycles can be actively controlled using PWM inverters [10]. This technique was named "active energy harvesting" by Liu et al. [35], and further analyzed for bloodpressure energy harvesting [36]. Such active principle theoretically enables to give any shape to the piezoelectric voltage waveform, including for instance the "optimal impedance emulation" described in section 3.1, yielding outstanding power level in theory. However, power consumption of the PWM control and energy losses of the circuit may limit the actual performances.

In summary, this subsection presented an overview of the main nonlinear circuits proposed until now. The reader will find more detailed analysis of these circuits in the original articles given in the References list. The next subsection presents a detailed analysis of the so-called OSECE circuit [37], which is one of the last proposed nonlinear circuits. It can be seen as a variant of the SSHI-MR circuit, but with simplified control principle, making much easier lowpower standalone implementation.

\subsection{The OSECE approach}

The OSECE (Optimized Synchronous Electrical Charge Extraction) has been developed as an improvement of the SECE approach. It allows to keep its main feature, namely the low dependency of the performance on the electrical load, while simplifying the electronic switches driving signals and enhancing the energy conversion [37]. 
The OSECE interface is shown in Figure 26. A transformer with two primary and one secondary windings divides this circuit into two parts: the left part is very similar to the SSDI circuit [19], including the switch control signal; the right part is similar to the secondary of a typical flyback DC/DC converter (smoothing capacitor $C_{r}$ plus equivalent load resistor $R_{L}$ ).

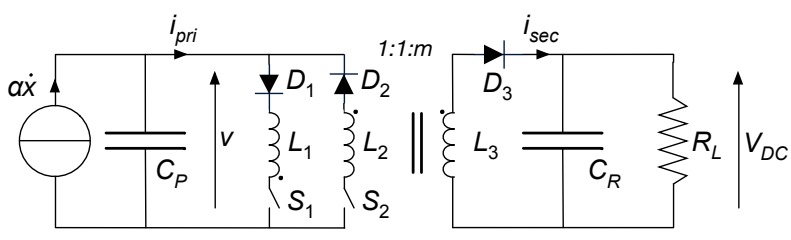

Figure 26. Electrical network using the OSECE strategy

The switch $S_{1}$ and $S_{2}$ are complementary driven: $S_{1}$ is closed and $S_{2}$ opened after the displacement (voltage) reaches a maximum and $S_{1}$ is opened and $S_{2}$ closed after the displacement (voltage) reaches a minimum. This switching strategy allows the voltage to be partly inverted two times a period of vibration.

The voltage is only partly inverted because the inversion phase is stopped as soon as the voltage on the secondary reaches $V_{D C}$ (at this moment, the diode in series with the closed switch becomes reversed biased). A detailed description and the modeling of this interface circuit can be found in [37].

Figure 27 shows the typical OSECE waveforms for the displacement, the piezoelectric voltage and the switch control signal for several periods. Details of voltages and currents close to the energy extraction moments are also shown. $V_{M}$ and $V_{m}$ are the piezoelectric voltage values just before and just after the energy extraction phase whose duration is $t_{m}$.

For the calculation of the performance of the OSECE approach, several assumptions are made: the magnetic circuit is linear; the coupling between the primary and secondary windings is ideal; the on-state voltage induced by the switches and the diodes are neglected; the output voltage $V_{D C}$ is ripple-free; and sinusoidal ambient accelerations around the resonance frequency of the PVEH are considered.

The value of $V_{D C}$ can be obtained as a function of the magnitude $x_{M}$ of the dynamic mass relative displacement:

$$
V_{D C}=-2 m \frac{\alpha}{C_{P}} \frac{\cos (\theta) e^{\frac{-\theta}{2 Q_{l}}}}{1+\cos (\theta) e^{\frac{-\theta}{Q_{l}}}} x_{M} \quad\left(V_{D C} \geq 0\right)
$$

where $Q_{I}$ is the quality factor of the primary $\left\{L_{1}, C_{P}\right\}$ oscillating circuit, $m$ is the turns ratio of the transformer, and $\theta$ is the phase angle corresponding to the duration of the inversion phase, given by:

$$
\theta=\pi-\arctan \left(m \sqrt{\frac{2 \pi}{R_{L} C_{P} \omega}}\right)=\pi-\arctan \left(m \sqrt{\frac{2 \pi}{r^{\prime} \Omega}}\right)
$$




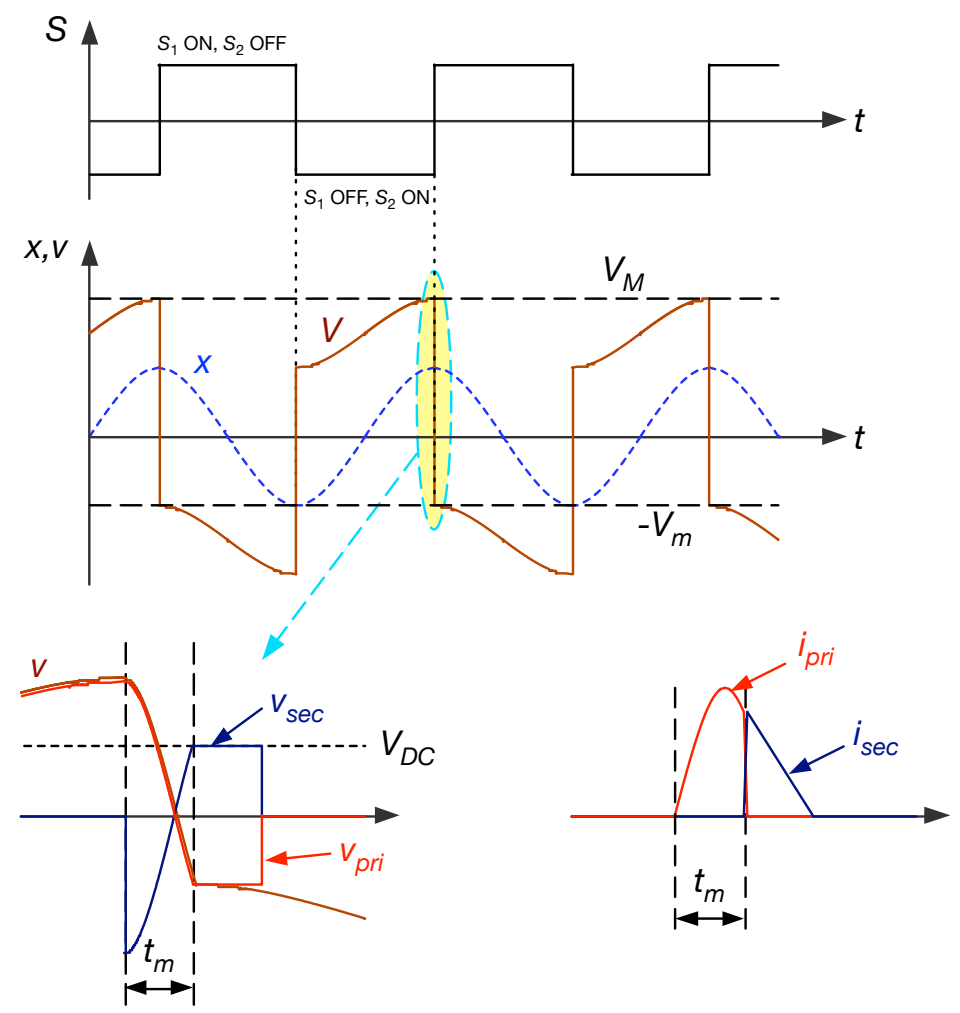

Figure 27. Typical waveforms for the OSECE approach (from [38])

As for the classical rectifier approach, only the fundamental component $v_{1}$ of the piezoelectric voltage, given by (4.4), is considered to impact the motion of the dynamic mass.

$$
\underline{v}_{1}=\frac{\alpha}{C_{P}}(v+j v) \underline{x} \quad \text { with }\left\{\begin{array}{l}
v=1 \\
v=\frac{4}{\pi} \frac{1-\cos (\theta) e^{\frac{-\theta}{2 Q_{1}}}}{1+\cos (\theta) e^{\frac{-\theta}{2 Q_{l}}}}
\end{array}\right.
$$

The expression in the frequency domain of the displacement and the normalized displacement are still the same as in section 3.1, equations (2.9) and (2.10) respectively.

Since $v=1$, the natural angular frequency of the PVEH is the open-circuit angular resonance frequency $\omega_{0} \sqrt{1+k_{m}^{2}}$. It can also be seen that $v$ is always larger that $4 / \pi$ since $\theta$ is between $\pi / 2$ and $\pi$ ) and depends on $\theta \quad Q_{I}$. Compared to the classical rectifier case where $v \quad \pi$ it is clear that the OSECE approach induces more damping.

As for the classical approach, the harvested power can be expressed as (3.5), and the normalized harvested power is finally given by:

$$
P^{\prime}=\frac{16}{\pi} k_{m}^{2} \frac{\Omega \sin (\theta) e^{-\frac{\theta}{Q_{l}}}}{\left(1+\cos (\theta) e^{-\frac{\theta}{2 Q_{l}}}\right)^{2}}\left|\underline{x}^{\prime}\right|^{2}
$$

The maximal normalized power and the corresponding optimal normalized load are plotted as a function of the frequency in Figure 28, for different values of $k_{m}^{2}$. When $k_{m}^{2}$ increases, it can be seen that whereas the bandwidth keeps on increasing, the maximal normalized power first 
increases and then decreases. This decrease is due to the too large damping effect induced by the OSECE approach in the case of highly coupled PVEH. Consequently comparing the power using the OSECE approach and the classical rectifier approach, it is found that OSECE leads to better performance for structures with low coupling coefficients or structures driven out of their resonance frequency.

The colored areas in the lower plot represent the load domains for which at least $50 \%$ of the maximal power is harvested. It is shown that the dependency on the load is much lower than when using the classical rectifier approach.
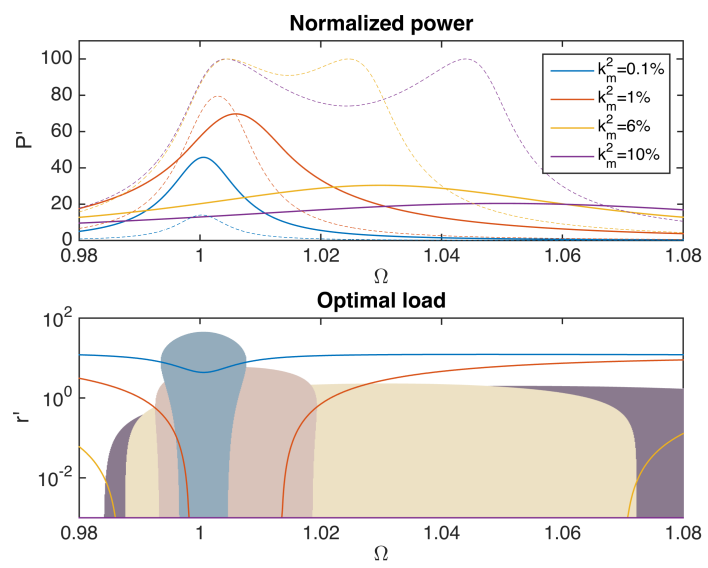

Figure 28. Upper plot: normalized power as a function of $\Omega$ for $\xi_{L}=0.005\left(Q_{M}=100\right)$ and different values of $k_{m}^{2}$ (plain line: OSECE, dashed line: simple rectifier). Lower plot: normalized optimal load as a function of $\Omega$ (areas corresponds to the load domain for which the power is at least $50 \%$ of the maximal power)

The normalized power, bandwidth and the figure of merit defined in (1.12) are plotted in Figure 29 as a function of $k_{m}^{2}$ for different values of $Q_{M}$. Results using the OSECE circuit are plotted as plain lines, and can be compared with results from the classical approach, which are plotted as dashed lines. Figure 29a shows that the power using the OSECE approach is larger when $k_{m}^{2} Q_{M}$ is lower than approximately $0.7\left(k_{m}^{2} Q_{M}=0.7\right.$ corresponds to the black curve). This particular value depends on $Q_{I}$, which was set to 5 in this calculation. Figure $29 \mathrm{~b}$ shows that the bandwidth using the OSECE approach is always larger, confirming the interest of this approach for PVEHs exited out of their resonances. Finally, Figure 29c shows that the figure of merit is higher using the OSECE approach when $k_{m}^{2} Q_{M}$ is lower than approximately $2.8\left(k_{m}^{2} Q_{M}=2.8\right.$ corresponds to the black line).

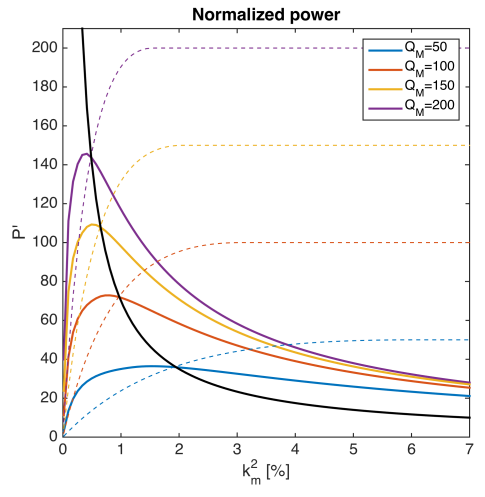

a

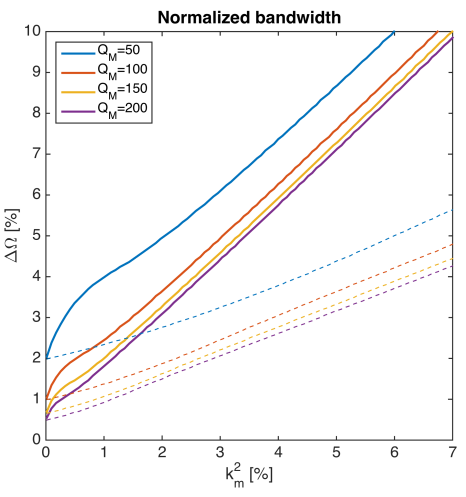

b

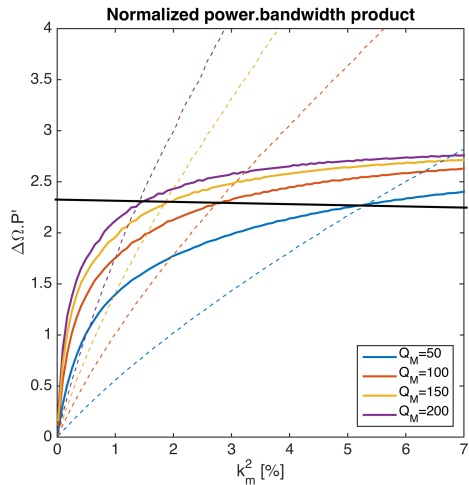

C

Figure 29. a) Normalized power versus $k_{m}^{2}$, b) normalized bandwidth versus $k_{m}^{2}$, c) FoM versus $k_{m}^{2}$ Plain lines: OSECE $\left(Q_{l}=5, m=1\right)$, dotted lines: simple rectifier 
It is worthy of note that the performances plotted in Figure 29 are given for the optimal electrical load. Practical implementations of the classical approach may lead to significantly poorer results than theoretical ones because of the strong dependency of the harvested power on the load, which imply the mandatory use of a complex MPPT (Maximum Power Point Tracking) strategy. On the other hand, the OSECE approach is much more tolerant to the load variations, which makes its practical performance closer from theory [38]. 


\section{ELECTRICAL FREQUENCY TUNING}

\subsection{The need for wideband PEH}

Most of PEHs reported in the literature exhibit squared coupling coefficient $k_{m}^{2}$ lower than $5 \%$. As shown in the previous sections, their bandwidth can be increased using nonlinear energy extraction circuits, but are still limited to a few percent of their resonance frequency. Such PEH can thus efficiently operate in a narrow frequency band tuned to match the excitation frequency.

However, environmental excitations have broadband or time-dependent characteristics in which the energy is distributed over a spread spectrum of frequencies. Two strategies have been investigated: Developing nonlinear wideband oscillators and developing linear oscillator with resonance frequency tuning mechanisms. Nonlinear oscillators (hardening, softening or bistable) are well suited for broadband vibration spectrum [39], whereas resonance frequency tuning is more appropriated for narrow band but time-dependent vibrations (which can be found on a motor whose rotation speed is varying). Nonlinear oscillators approaches are beyond the scope of this chapter since they are not related to the energy extraction circuit but to the architecture of the PEH. Resonance frequency tuning is usually done through an additional mechanical component that passively or actively changes the stiffness or inertia of a linear mechanical oscillator [40]. These mechanical approaches will also not be detailed here.

This section reports a theoretical nonlinear energy extraction approach to tune the resonant frequency of linear inertial PEH through the control strategy of the associated electronic interface circuit. When associated with piezoelectric devices exhibiting high electromechanical coupling, it enables to vary the resonant frequency in large proportions without any additional component.

Seddik et al previously proposed a principle of control through the electronic interface circuit [41]. It consisted in connecting shunt capacitors to the piezoelectric device. Consequently, the electromechanical structure stiffness was varied, and the resonant frequency was changed accordingly. They showed that it was possible to significantly vary the resonant frequency of highly coupled piezoelectric energy harvesters. However, because the shunt capacitance was varied step by step, the resonant frequency could not be continuously tuned. With the proposed technique, it is expected that a continuous tuning is achievable on a noticeably larger frequency range.

\subsection{Frequency Tuning SECE (FTSECE)}

The FTSECE approach is also derived from the previously developed SECE (Synchronized Electrical Charge Extraction) approach. It is called FTSECE for Frequency Tuning SECE [42]. It consists in letting the PEH in open circuit condition most of the time, and to extract the generated electrical charges two times a period of vibration. In contrast to the SECE technique, the FTSECE circuit does not extract energy at the piezoelectric voltage extremum, but with a phase shift $\phi$. Moreover, whereas the piezoelectric voltage is null after each energy extraction phase in the SECE approach (all the electrical charges are extracted), it can be tuned with the FTSECE technique.

The FTSECE approach has not been practically demonstrated yet, but a possible electronic interface circuit for its realization is schematically depicted in Figure 30. This circuit includes an electronic switch $S$, whose control is synchronized with the piezoelectric voltage. Two parameters of $S$ are varied through the control circuit: the on state time duration $\left(t_{O N}\right)$ and the lag time duration $\left(t_{\text {lag }}\right)$ between the voltage extrema and the instant where $S$ is turned on. The phase shift $\phi=\omega t_{\text {lag }}$ can be positive ( $S$ is closed after the voltage extremum) or negative ( $S$ is closed before the voltage extremum). If $V_{M}$ is the piezoelectric voltage at the moment where $S$ is turned on, varying $t_{O N}$ will allow to set the voltage to $\beta V_{M}$ after the energy extraction phase. If $Q_{I}$ is the quality factor of the $\left\{L, C_{P}\right\}$ circuit, $\beta$ $\left(t_{O N} \simeq \pi\left(L C_{P}\right)^{1 / 2}\right)$ be tuned between $1\left(t_{O N}=0\right)$ and $-e^{-\pi /\left(2 Q_{I}\right)}$ 
The load resistance value has no influence on the harvested power provided that the DC load voltage is larger than the piezoelectric voltage amplitude. In this way, energy transfers from the inductor to the rectifier only occur right after $S$ is turned off. Varying $t_{O N}$ enables to tune the amount of electrical energy extracted from the piezoelectric elements, and, if needed, to reverse the polarity of the piezoelectric voltage. Varying $t_{\text {lag }}$ enables to modify the electromechanical structure stiffness by tuning the phase shift between the piezoelectric voltage and the strain in the piezoelectric element.

Typical waveforms for the FTSECE approach are given in Figure 31. Using the same procedure as in previous sections, the fundamental component $v_{1}$ of the piezoelectric voltage is calculated:

$$
\underline{v}_{1}=\frac{\alpha}{C_{P}}(v+j v) \underline{x} \text { with }\left\{\begin{aligned}
v & =1+\frac{2}{\pi} \frac{1-\beta}{1+\beta} \sin (2 \phi) \\
v & =\frac{4}{\pi} \frac{1-\beta}{1+\beta} \cos ^{2}(\phi)
\end{aligned}\right.
$$

Equations (5.1) confirm that $\phi$ and $\beta$ ( $t_{\text {lag }}$ and $t_{0 N}$ ) affect both the resonance frequency of the system and its damping. Frequency tuning as well as optimization of the energy transfer is then achievable using the FTSECE approach.

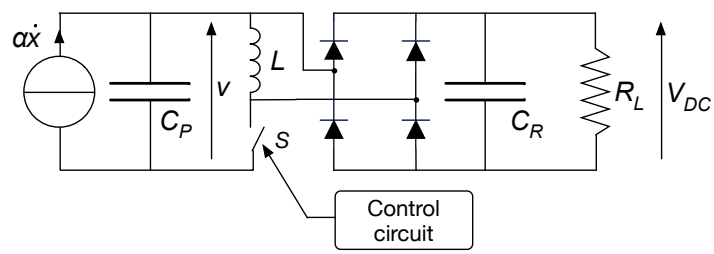

Figure 30 Schematic representation of the FTSECE electronic interface circuit

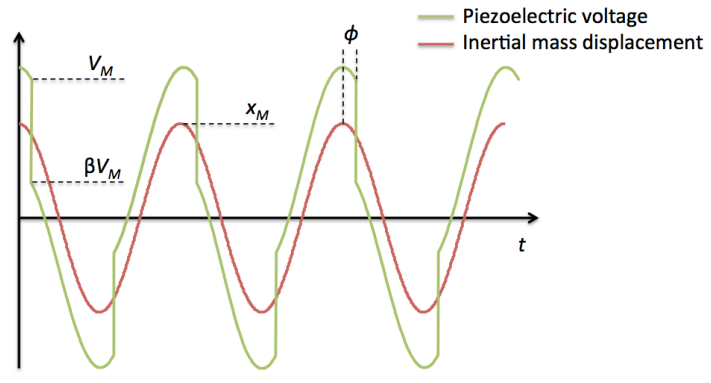

Figure 31 Typical waveforms for the FTSECE approach

The power extracted from the PEH is calculated from the energy extracted two times a period of the ambient vibration:

$$
P_{E X}=\frac{\omega}{2 \pi} C_{P} V_{M}^{2}\left(1-\beta^{2}\right)
$$

The expression of $V_{M}$ is given by (5.3) where $x_{M}$ is the magnitude of the dynamic mass displacement.

$$
V_{M}=\frac{\alpha}{C_{P}} x_{M} \cos (\phi) \frac{2}{1+\beta}
$$

Taking into account the losses in the inductor $L$ the normalized harvested power can then be approximated by:

$$
P^{\prime}=\frac{16}{\pi} k_{m}^{2} \Omega \cos ^{2}(\phi) \frac{(1-\beta)\left(e^{-\pi /\left(2 Q_{I}\right)}+\beta\right)}{(1+\beta)^{2}}\left|\underline{x}^{\prime}\right|^{2}
$$

The normalized power as well as the optimal $\phi$ and $\beta$ are plotted in Figure 32 as a function of the operating frequency, for different values of $k_{m}^{2}$. High coupling coefficients have been considered because the FTSECE approach is especially promising in this case. Such coupling coefficients can be practically obtained using single crystals piezoelectric materials. For instance, a PEH for which $k^{2}$ equals $53 \%\left(k_{m}^{2}=112 \%\right)$ was presented in [42]. 
From the evolution of $\phi$ and $\beta$, it can be seen that the extraction times coincide with the voltage extrema $(\phi=0)$ only at the open-circuit resonance frequency of the PEH. In this case, if the coupling coefficient is high (typically larger than 5\%), only a small amount of the generated electrical charges are extracted ( $\beta$ tends to 1$)$ to prevent a too large damping of the PEH. When the vibration frequency is slightly shifted away from the open-circuit resonance frequency, the switching phase shift $\phi$ is adjusted to tune the resonance frequency of the PEH. This phase shift induces a decrease of the generated electrical charges, which is compensated by extracting a larger percentage of them. If the vibration frequency is shifted further, $\beta$ becomes negative, which means that a portion of the generated electrical charges is injected back to the PEH with a reversed polarity. This effect increases the piezoelectric voltage amplitude and enhances the energy extraction.
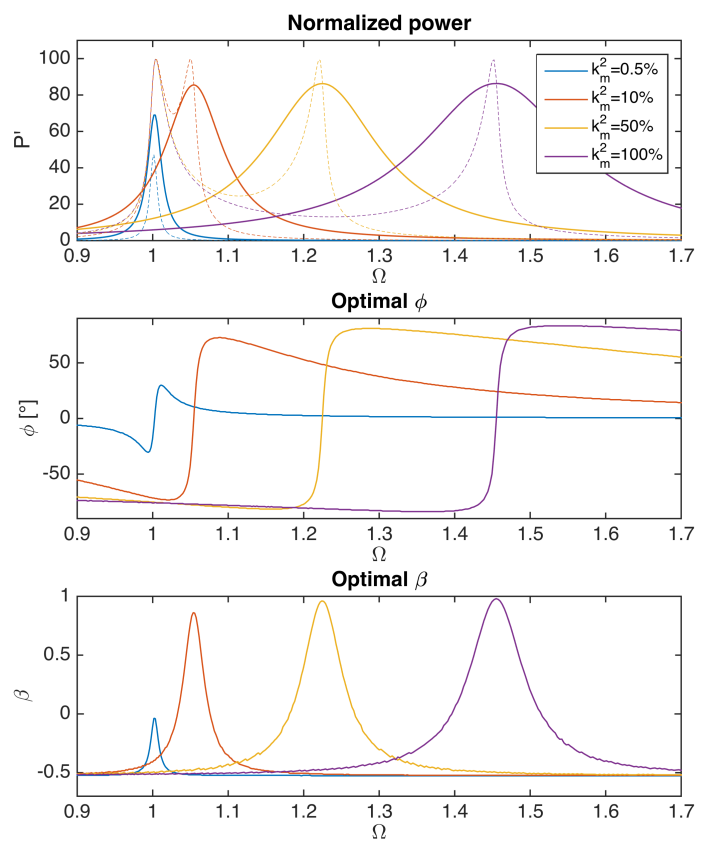

Figure 32. Upper plot: normalized power as a function of $\Omega$ for $\xi_{L}=0.005\left(Q_{M}=100\right)$ and different values of $k_{m}^{2}$ (plain line: FTSECE, dashed line: simple rectifier). Middle plot: optimal $\phi$ as a function of $\Omega$. Lower plot: optimal $\beta$ as a function of $\Omega$.

The normalized power, bandwidth and the figure of merit are plotted in Figure 33 as a function of $k_{m}^{2}$ for different values of $Q_{M}$. Results using the FTSECE circuit are plotted as plain lines, and can be compared with results from the classical approach plotted as dashed lines.

Figure 33a shows that comparing to the classical rectifier approach the power using the FTSECE approach is larger when $k_{m}^{2} Q_{M}<0.9$ and slightly lower when $k_{m}^{2} Q_{M}>0.9$ (the black curve corresponds to $k_{m}^{2} Q_{M}=0.9$ ). Figure 33b confirms the bandwidth enhancement induced by the FTSECE circuit. Finally, Figure 33c shows that the FTSECE approach gives higher figure of merit whatever the value of $k_{m}^{2}$.

The FTSECE circuit has not been practically implemented yet. The real-time tuning of two parameters $\left(t_{\text {on }}\right.$ and $\left.t_{\text {lag }}\right)$ requires the development of a dedicated switch control circuit, whose complexity may hinder the overall performances. Moreover, the effect of the piezoelectric voltage switching on highly coupled PEH may generate non-sinusoidal displacement of the dynamic mass, in which case the theoretical calculation detailed above become inaccurate. Because of the lack of experimental demonstration, this last section should then be considered with caution. It however suggests that resonant piezoelectric structures with high 
electromechanical coupling coefficient combined with dedicated nonlinear energy extraction circuit could lead to large bandwidth PEH (up to $20 \%$ of the resonance frequency could reasonably be obtained).

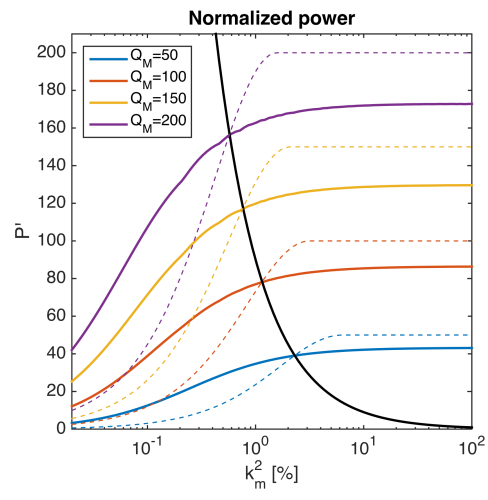

a

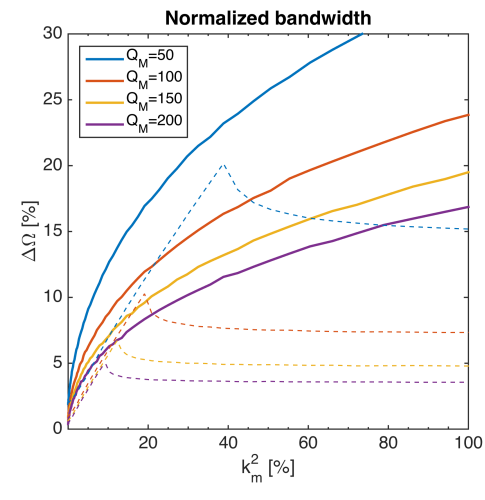

b

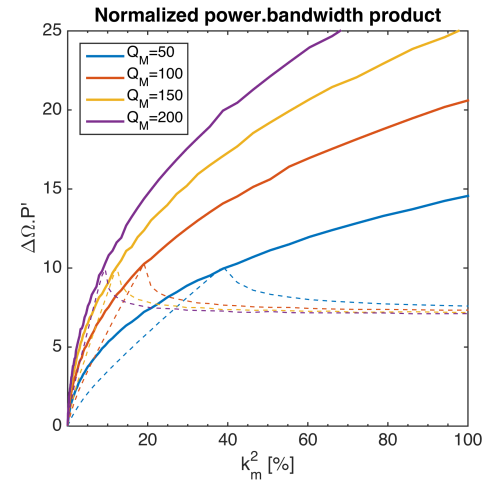

C

Figure 33. a) Normalized power versus $k_{m}^{2}$, b) normalized bandwidth versus $k_{m}^{2}$, c) FoM versus $k_{m}^{2}$ Plain lines: FTSECE $\left(Q_{I}=5, m=1\right)$, dotted lines: simple rectifier

To compare the FTSECE approach and the ideal impedance matching strategy (cf. section 2), it is assumed that $\xi_{E}=0.005$. Taking $k_{m}^{2}=100 \%$ and $\xi_{M}=0.005\left(Q_{M}=100\right)$ leads to $\chi$ . The FoM using the impedance matching strategy would then be $\sqrt{\chi}=200$, whereas it is only 20 using FTSECE (see red curve at $k_{m}^{2}=100 \%$ in Figure 33c). This result confirms that the ideal impedance matching gives an upper limit for a PVEH performance. It also suggests that some advanced energy harvesting circuits with enhanced bandwidth capability might be developed by further research. 


\section{CONCLUSION}

This chapter presents a broad analysis of the existing power conditioning techniques for piezoelectric energy harvesting devices and the related circuits enabling practical implementation.

The classical impedance matching technique would enable in theory to get the best performances in terms of power and frequency bandwidth. However, practical implementation of this technique would require complicated, power consuming control algorithms. To date, very few experimental results can be found about this technique [8], confirming difficult implementation.

Overall, the so-called nonlinear interfaces bring several advantages. One of their most remarkable properties is the drastic power improvement of PVEH exhibiting low $k^{2} Q_{m}$. For PVEH with higher $k^{2} Q_{m}$, they give enhanced performance for pulsed excitation or out of resonance excitation.

Another advantage is that some of these nonlinear interfaces, such as SECE and OSECE, tend to minimize the effect of the electrical load on the energy conversion, which is a very interesting property when supplying a device with time varying electrical characteristics.

Finally, nonlinear interfaces, such as the FTCECE, may be used to electronically tune the PVEH resonant frequency, which could be used to drastically enhance their bandwidth.

Current trends in the domain of power conditioning circuits for piezoelectric vibration energy harvesting tend to push the limits towards "high" voltages (above $50 \mathrm{~V}$ ) and "low" voltages (below $1 \mathrm{~V}$ ) with ultra-low harvested power, typically in the range of $10 \mathrm{nW}$ to $10 \mu \mathrm{W}$. Such developments raise new challenges in the domain of ultra-low power AC-DC and DC-DC converters, and in the design of ultra-low power ASICs. 
[1] C. B. Williams and R. B. Yates, "Analysis of a micro-electric generator for microsystems," Sensors and Actuators A: Physical, vol. 52, no. 1, pp. 8-11, Mar. 1996.

[2] E. Arroyo, A. Badel, F. Formosa, Y. P. Wu, and J. Qiu, "Comparison of electromagnetic and piezoelectric vibration energy harvesters: Model and experiments," Sensors and Actuators A: Physical, vol. 183, pp. 148-156, Aug. 2012.

[3] R. Krimholtz, D. A. Leedom, and G. L. Matthaei, "New equivalent circuits for elementary piezoelectric transducers," Electronics Letters, vol. 6, no. 13, pp. 398-399, 1970.

[4] H. A. C. Tilmans, "Equivalent circuit representation of electromechanical transducers: I. Lumped-parameter systems," J. Micromech. Microeng., vol. 6, no. 1, pp. 157-176, Mar. 1996.

[5] Y. Yang and L. Tang, "Equivalent Circuit Modeling of Piezoelectric Energy Harvesters," JIMSS, vol. 20, no. 18, pp. 2223-2235, Nov. 2009.

[6] J. M. Renno, M. F. Daqaq, and D. J. Inman, "On the optimal energy harvesting from a vibration source," JSV, vol. 320, no. 1, pp. 386-405, Feb. 2009.

[7] D. Guyomar, A. Badel, E. Lefeuvre, and C. Richard, "Toward energy harvesting using active materials and conversion improvement by nonlinear processing," Ultrasonics, Ferroelectrics, and Frequency Control, IEEE Transactions on, vol. 52, no. 4, pp. 584-595, Apr. 2005.

[8] Y. Li and C. Richard, "Piezogenerator impedance matching using Mason equivalent circuit for harvester identification," presented at the SPIE Smart Structures and Materials + Nondestructive Evaluation and Health Monitoring, 2014, vol. 9057, pp. 90572I-13.

[9] R. W. Erickson and D. Maksimovic, Fundamentals of Power Electronics. Springer US, 2001.

[10] E. Lefeuvre, G. Sebald, D. Guyomar, and M. Lallart, "Materials, structures and power interfaces for efficient piezoelectric energy harvesting," Journal of ..., 2009.

[11] Y. C. Shu and I. C. Lien, "Analysis of power output for piezoelectric energy harvesting systems," Smart Mater. Struct., vol. 15, no. 6, pp. 1499-1512, Sep. 2006.

[12] G. K. Ottman, H. F. Hofmann, A. C. Bhatt, and G. A. Lesieutre, "Adaptive piezoelectric energy harvesting circuit for wireless remote power supply," Power Electronics, IEEE Transactions on, vol. 17, no. 5, pp. 669-676, Sep. 2002.

[13] G. K. Ottman, H. F. Hofmann, and G. A. Lesieutre, "Optimized piezoelectric energy harvesting circuit using step-down converter in discontinuous conduction mode," Power Electronics, IEEE Transactions on, vol. 18, no. 2, pp. 696-703, Mar. 2003.

[14] E. Lefeuvre, D. Audigier, C. Richard, and D. Guyomar, "Buck-Boost Converter for Sensorless Power Optimization of Piezoelectric Energy Harvester," Power Electronics, IEEE Transactions on, vol. 22, no. 5, pp. 2018-2025, 2007.

[15] J. Yi, F. Su, Y.-H. Lam, W.-H. Ki, and C.-Y. Tsui, "An energy-adaptive MPPT power management unit for micro-power vibration energy harvesting," presented at the 2008 IEEE International Symposium on Circuits and Systems - ISCAS 2008, 2008, pp. 25702573.

[16] N. Kong and D. S. Ha, "Low-Power Design of a Self-powered Piezoelectric Energy Harvesting System With Maximum Power Point Tracking," Power Electronics, IEEE Transactions on, vol. 27, no. 5, pp. 2298-2308, May 2012.

[17] M. Shim, J. Kim, J. Jung, and C. Kim, "Self-powered 30 $\mu$ W-to-10mW Piezoelectric energyharvesting system with $9.09 \mathrm{~ms} / \mathrm{V}$ maximum power point tracking time," 2014 IEEE International Solid-State Circuits Conference (ISSCC), pp. 406-407, 2014.

[18] S. Bandyopadhyay, P. P. Mercier, A. C. Lysaght, K. M. Stankovic, and A. P. Chandrakasan, "A 1.1 nW Energy-Harvesting System with 544 pW Quiescent Power for NextGeneration Implants," IEEE Journal of solid-state circuits, vol. 49, no. 12, pp. 2812-2824, Dec. 2014.

[19] C. Richard, D. Guyomar, D. Audigier, and G. Ching, "Semi-passive damping using continuous switching of a piezoelectric device," presented at the 1999 Symposium on Smart Structures and Materials, 1999, vol. 3672, pp. 104-111. 
[20] A. Badel, "Efficiency Enhancement of a Piezoelectric Energy Harvesting Device in Pulsed Operation by Synchronous Charge Inversion," JIMSS, vol. 16, no. 10, pp. 889-901, Oct. 2005.

[21] E. Lefeuvre, A. Badel, C. Richard, and D. Guyomar, "High-performance piezoelectric vibration energy reclamation," presented at the Smart Structures and Materials, 2004, vol. 5390, pp. 379-387.

[22] Y. C. Shu, I. C. Lien, and W. J. Wu, "An improved analysis of the SSHI interface in piezoelectric energy harvesting," Smart Mater. Struct., vol. 16, no. 6, pp. 2253-2264, Oct. 2007.

[23] E. Lefeuvre, A. Badel, C. Richard, L. Petit, and D. Guyomar, "A comparison between several vibration-powered piezoelectric generators for standalone systems," Sensors and Actuators A: Physical, vol. 126, no. 2, pp. 405-416, Feb. 2006.

[24] G. W. Taylor, J. R. Burns, S. M. Kammann, W. B. Powers, and T. R. Wel, "The Energy Harvesting Eel: a small subsurface ocean/river power generator," Oceanic Engineering, IEEE Journal of, vol. 26, no. 4, pp. 539-547, Oct. 2001.

[25] A. Badel, A. Benayad, E. Lefeuvre, L. Lebrun, C. Richard, and D. Guyomar, "Single crystals and nonlinear process for outstanding vibration-powered electrical generators," IEEE Transactions on Ultrasonics, Ferroelectrics and Frequency Control, vol. 53, no. 4, pp. 673684, 2006.

[26] E. Lefeuvre, "Piezoelectric Energy Harvesting Device Optimization by Synchronous Electric Charge Extraction," JIMSS, vol. 16, no. 10, pp. 865-876, Oct. 2005.

[27] K. Makihara, J. Onoda, and T. Miyakawa, "Low energy dissipation electric circuit for energy harvesting," Smart Mater. Struct., vol. 15, no. 5, pp. 1493-1498, Sep. 2006.

[28] M. Lallart and D. Guyomar, "An optimized self-powered switching circuit for non-linear energy harvesting with low voltage output," Smart Mater. Struct., vol. 17, no. 3, pp. 035030-9, May 2008.

[29] L. Garbuio, M. Lallart, D. Guyomar, C. Richard, and D. Audigier, "Mechanical Energy Harvester With Ultralow Threshold Rectification Based on SSHI Nonlinear Technique," Industrial Electronics, IEEE Transactions on, vol. 56, no. 4, pp. 1048-1056, 2009.

[30] A. D. T. Elliott and P. D. Mitcheson, "Implementation of a Single Supply Pre-biasing Circuit for Piezoelectric Energy Harvesters," Procedia Engineering, vol. 47, pp. 13111314, 2012.

[31] M. Lallart, L. Garbuio, L. Petit, C. Richard, and D. Guyomar, "Double synchronized switch harvesting (DSSH): a new energy harvesting scheme for efficient energy extraction," Ultrasonics, Ferroelectrics, and Frequency Control, IEEE Transactions on, vol. 55, no. 10, pp. 2119-2130, Sep. 2008.

[32] H. Shen, J. Qiu, H. Ji, K. Zhu, and M. Balsi, "Enhanced synchronized switch harvesting: a new energy harvesting scheme for efficient energy extraction," Smart Mater. Struct., vol. 19, no. 11, p. 115017, Sep. 2010.

[33] W. J. Wu, A. M. Wickenheiser, T. Reissman, and E. Garcia, "Modeling and experimental verification of synchronized discharging techniques for boosting power harvesting from piezoelectric transducers," Smart Mater. Struct., vol. 18, no. 5, pp. 055012-15, Apr. 2009.

[34] J. Dicken, P. D. Mitcheson, and I. Stoianov, "Increased power output from piezoelectric energy harvesters by pre-biasing," presented at the PowerMEMS 2011, 2009.

[35] Yiming Liu, Geng Tian, Yong Wang, Junhong Lin, Qiming Zhang, and H. F. Hofmann, "Active Piezoelectric Energy Harvesting: General Principle and Experimental Demonstration," JIMSS, vol. 20, no. 5, pp. 575-585, Mar. 2009.

[36] M. Deterre, E. Lefeuvre, and E. Dufour-Gergam, "An active piezoelectric energy extraction method for pressure energy harvesting," Smart Mater. Struct., vol. 21, no. 8, p. 085004, Jul. 2012.

[37] Y. P. Wu, A. Badel, F. Formosa, W. Q. Liu, and A. E. Agbossou, "Piezoelectric vibration energy harvesting by optimized synchronous electric charge extraction," JIMSS, vol. 24, no. 12, pp. 1445-1458, Jul. 2013. 
[38] Y. P. Wu, A. Badel, F. Formosa, W. Q. Liu, and A. Agbossou, "Self-powered optimized synchronous electric charge extraction circuit for piezoelectric energy harvesting," JIMSS, vol. 25, no. 17, pp. 2165-2176, Oct. 2014.

[39] L. Gammaitoni, I. Neri, and H. Vocca, "Nonlinear oscillators for vibration energy harvesting," Appl. Phys. Lett., vol. 94, no. 16, p. 164102, Apr. 2009.

[40] C. Eichhorn, R. Tchagsim, N. Wilhelm, and P. Woias, "A smart and self-sufficient frequency tunable vibration energy harvester," J. Micromech. Microeng., vol. 21, no. 10, p. 104003, Sep. 2011.

[41] B. Ahmed Seddik, G. Despesse, and E. Defay, "Autonomous wideband mechanical energy harvester," presented at the Industrial Electronics ISIE, IEEE International Symposium on, Hangzhou, China, 2012.

[42] A. Badel and E. Lefeuvre, "Wideband Piezoelectric Energy Harvester Tuned Through its Electronic Interface Circuit," J. Phys.: Conf. Ser., vol. 557, p. 012115, Dec. 2014. 